\title{
Quantum Versus Classical Spin Fragmentation in Dipolar Kagome Ice $\mathrm{Ho}_{3} \mathrm{Mg}_{2} \mathrm{Sb}_{3} \mathrm{O}_{14}$
}

\author{
Zhiling Dun $\odot,{ }^{1,2, *, \|}$ Xiaojian Bai, ${ }^{1, \|}$ Joseph A. M. Paddison, ${ }^{1,3,4, \dagger}$ Emily Hollingworth, ${ }^{1}$ Nicholas P. Butch, ${ }^{5}$ \\ Clarina D. Cruz, ${ }^{6}$ Matthew B. Stone, ${ }^{6}$ Tao Hong, ${ }^{6}$ Franz Demmel, ${ }^{7}$ Martin Mourigal, ${ }^{1, \$}$ and Haidong Zhou ${ }^{2,8,8}$ \\ ${ }^{1}$ School of Physics, Georgia Institute of Technology, Atlanta, Georgia 30332, USA \\ ${ }^{2}$ Department of Physics and Astronomy, University of Tennessee, Knoxville, Tennessee 37996, USA \\ ${ }^{3}$ Churchill College, University of Cambridge, Storey's Way, Cambridge CB3 ODS, United Kingdom \\ ${ }^{4}$ Materials Science and Technology Division, Oak Ridge National Laboratory, \\ Oak Ridge, Tennessee 37831, USA \\ ${ }^{5}$ NIST Center for Neutron Research, Gaithersburg, Maryland 20899, USA \\ ${ }^{6}$ Neutron Scattering Division, Oak Ridge National Laboratory, Oak Ridge, Tennessee 37831, USA \\ ${ }^{7}$ ISIS Facility, Rutherford Appleton Laboratory, Didcot OX11 0QX, United Kingdom \\ ${ }^{8}$ National High Magnetic Field Laboratory, Florida State University, Tallahassee, Florida 32310, USA
}

(Received 24 December 2019; revised 24 June 2020; accepted 31 July 2020; published 29 September 2020)

A promising route to realize entangled magnetic states combines geometrical frustration with quantumtunneling effects. Spin-ice materials are canonical examples of frustration, and Ising spins in a transverse magnetic field are the simplest many-body model of quantum tunneling. Here, we show that the tripodkagome lattice material $\mathrm{Ho}_{3} \mathrm{Mg}_{2} \mathrm{Sb}_{3} \mathrm{O}_{14}$ unites an icelike magnetic degeneracy with quantum-tunneling terms generated by an intrinsic splitting of the $\mathrm{Ho}^{3+}$ ground-state doublet, which is further coupled to a nuclear spin bath. Using neutron scattering and thermodynamic experiments, we observe a symmetrybreaking transition at $T^{*} \approx 0.32 \mathrm{~K}$ to a remarkable state with three peculiarities: a concurrent recovery of magnetic entropy associated with the strongly coupled electronic and nuclear degrees of freedom; a fragmentation of the spin into periodic and icelike components; and persistent inelastic magnetic excitations down to $T \approx 0.12 \mathrm{~K}$. These observations deviate from expectations of classical spin fragmentation on a kagome lattice, but can be understood within a model of dipolar kagome ice under a homogeneous transverse magnetic field, which we survey with exact diagonalization on small clusters and mean-field calculations. In $\mathrm{Ho}_{3} \mathrm{Mg}_{2} \mathrm{Sb}_{3} \mathrm{O}_{14}$, hyperfine interactions dramatically alter the single-ion and collective properties, and suppress possible quantum correlations, rendering the fragmentation with predominantly single-ion quantum fluctuations. Our results highlight the crucial role played by hyperfine interactions in frustrated quantum magnets and motivate further investigations of the role of quantum fluctuations on partially ordered magnetic states.

DOI: 10.1103/PhysRevX.10.031069

\section{INTRODUCTION}

Quantum spin liquids are exotic states of magnetic matter in which conventional magnetic order is suppressed by strong quantum fluctuations [1]. Frustrated magnetic materials, which have a large degeneracy of classical

\footnotetext{
*zdun3@gatech.edu

paddisonja@ornl.gov

*mourigal@gatech.edu

§hzhou10@utk.edu

"Z. D. and X. B. contributed equally to this work.
}

Published by the American Physical Society under the terms of the Creative Commons Attribution 4.0 International license. Further distribution of this work must maintain attribution to the author(s) and the published article's title, journal citation, and DOI.
Subject Areas: Condensed Matter Physics, Magnetism, Quantum Physics magnetic ground states, are often good candidates to search for this elusive behavior. A canonical example of frustration is spin ice, in which Ising spins occupy a pyrochlore lattice of corner-sharing tetrahedra [2,3]. Classical ground states obey the "two in, two out" ice rule for spins on each tetrahedron, and thermal excitations behave as deconfined magnetic monopoles [4-7]. These pairs of fractionalized excitations interact via Coulomb's law and correspond to topological defects of a classical field theory obtained by coarse graining spins into a continuous magnetization. In principle, topological quantum excitations can be generated by adding quantum-tunneling terms to the classical spinice model-e.g., by adding couplings between the transverse components of spins [8-10], or by introducing a local magnetic field transverse to the Ising spins [11-14]. A search for real materials that realize such quantum spin-ice states has found several promising candidates 


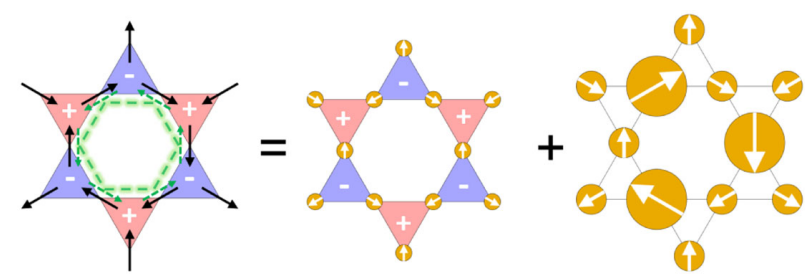

FIG. 1. Classical spin fragmentation (CSF) process in a model of dipolar kagome ice displaying emergent-charge order, based on Ref. [39]. The expectation values of spins $\left\langle\sigma_{i}^{z}\right\rangle$ are represented by black arrows. Each triangle has one spin pointing "in" (toward its center) and two pointing "out" (away from its center), or vice versa. The emergent magnetic charge of a triangle is defined as the number of spins pointing in minus the number pointing out. Positive $\left(Q_{j}=+\right)$ and negative $\left(Q_{j}=-\right)$ emergent charges are represented as red and blue triangles, respectively, and form a staggered arrangement. Three distinct spin configurations are possible for a given emergent charge, which yields a macroscopic number of degenerate spin configurations associated with emergent-charge ordering. Spin fragmentation decomposes each unitlength spin into "divergence-full" and "divergence-free" channels (center and right-hand images, respectively). The fragmented spins are shown as orange circles with diameter proportional to the length of the fragmented spin. The green hexagon represents the flipping of six spins around a closed loop: this is the simplest process that connects two distinct spin configurations within the degenerate CSF manifold.

(see, e.g., Refs. [15-25]). However, important challenges remain, including the determination of the often-complex spin Hamiltonian [26-28], the subtle role that structural disorder may play [29-31], and the computational challenges associated with simulations of three-dimensional (3D) quantum magnets $[32,33]$.

A promising alternative route toward quantum analogs of spin ice is offered by two-dimensional Ising ferromagnets on a kagome lattice. When the spins are confined to point either toward or away from the center of each triangle of the lattice, a highly degenerate kagome ice state is stabilized with a "one in, two out" or "two in, one out" local ice rule on each triangle [34]. Both a quantum-tunneling term and an external magnetic field are required to enable tunneling between these states $[35,36]$. Remarkably, when the longrange magnetic dipole-dipole interaction is introduced, the effective Coulomb interaction between emergent magnetic charges - defined in Fig. 1-selects a subspace of the kagome ice manifold and drives a phase transition to an intermediate-temperature phase with staggered emergentcharge ordering [37,38]. This state possesses nonzero entropy because each emergent charge retains a threefold degeneracy of spin orientations [37]; hence, ordering of the emergent charges does not imply complete long-range ordering of the spins. Figure 1 shows that such spin structures can be decomposed into a "divergence-full" channel in which spins are spatially ordered and an "divergence-free" channel in which spins remain spatially disordered-a process known as spin fragmentation [39,40].
Neutron-scattering measurements provide a direct experimental signature of spin fragmentation via the coexistence of magnetic Bragg peaks and highly structured magnetic diffuse scattering with pinch-point singularities [21,41,42]. The divergence-full channel corresponds to an "all-in, allout" (AIAO) order of fragmented spins that reflects the longrange staggered arrangement of emergent charges. In the divergence-free channel, fragmented spins are disordered but correlated and the constraint that every triangle has zero emergent charge yields a Coulomb phase analogous to pyrochlore spin ices [40]. Ultimately, similar to the pyrochlore spin ices, the dipolar interaction is expected to drive the system to a complete spin order at low temperature [37,38], although such thermally equilibrated states are generally not realized experimentally in real systems.

Without additional quantum effects, the classical spinfragmented (CSF) state described above can be viewed as a classical spin liquid coexisting with magnetic order. Its elementary excitations are thermally flipped spins that map onto pairs of magnetic monopoles, i.e., defects in the divergence-free channel. Typically, such thermally activated excitations are exponentially suppressed at low temperature, as observed experimentally in the CSF phase of the kagome ice compound $\mathrm{Dy}_{3} \mathrm{Mg}_{2} \mathrm{Sb}_{3} \mathrm{O}_{14} \quad[41,43]$. The question arises as to the effect of quantum fluctuations on degenerate classical spin configurations and whether a distinct phase of matter can be stabilized through quantum superposition, in close analogy to the quantum ice physics proposed for pyrochlore and square-lattice systems $[8-10,12,14,44]$. For instance, quantum tunneling can connect different CSF configurations through the concurrent flipping of six spins, a process shown with a green hexagon in Fig. 1. Conceptually, such dynamics may stabilize a putative "quantum spin fragmented" (QSF) state that remains characterized by the coexistence of Bragg peaks and highly structured diffuse scattering in magnetic neutron scattering experiments, but with dynamic magnetic correlations reflecting coherent collective excitations, akin to the emergent monopole and photonlike excitations in quantum spin ice $[10,45]$. It is unclear if such a partially disordered quantum state can theoretically prevail as an extended phase at finite temperature. Indeed, the previously studied transverse-field Ising model on the kagome lattice maps onto a quantum dimer model for which cooperative quantum fluctuations exist only at a finely tuned RokhsarKivelson point [46-48]. Furthermore, the dipolar kagome ice model shows a robust tendency toward magnetic ordering at low temperatures [38], even when a small transverse field is included [49].

This rich theoretical landscape motivates comprehensive experimental investigation of dipolar kagome ice materials. In real systems, details of the magnetic Hamiltonian going beyond the necessarily simplified aforementioned theoretical models will inevitably come into play. Some features, such as effective dimensionality of the magnetic interactions or subleading terms, may provide opportunities to 
(a)

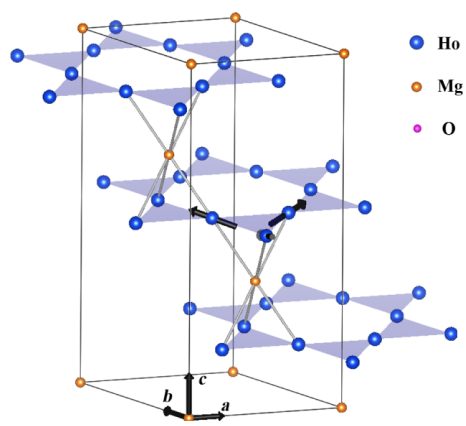

(d)

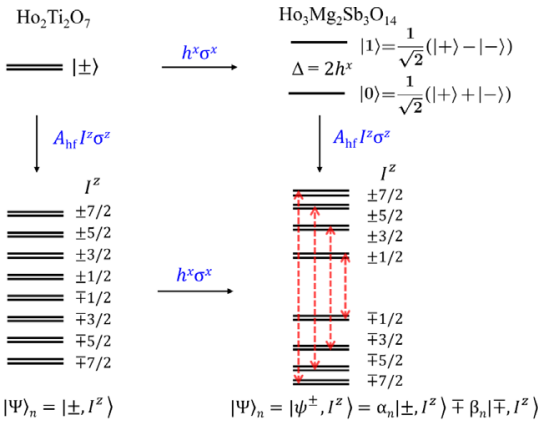

(b)

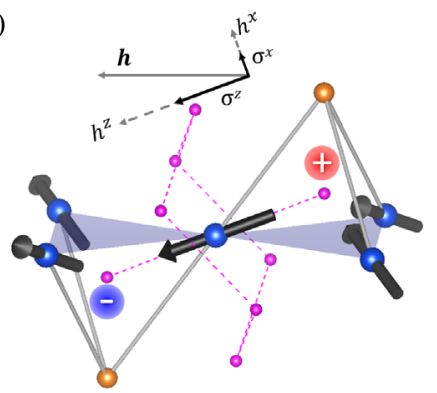

(e)

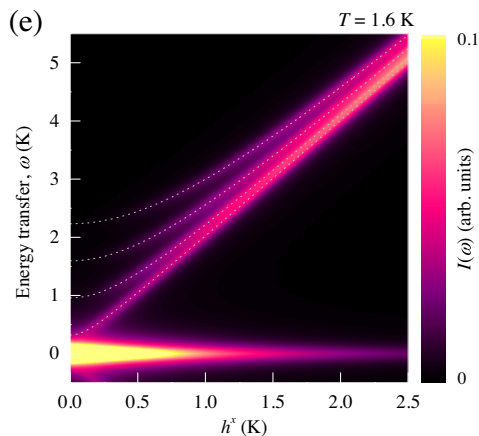

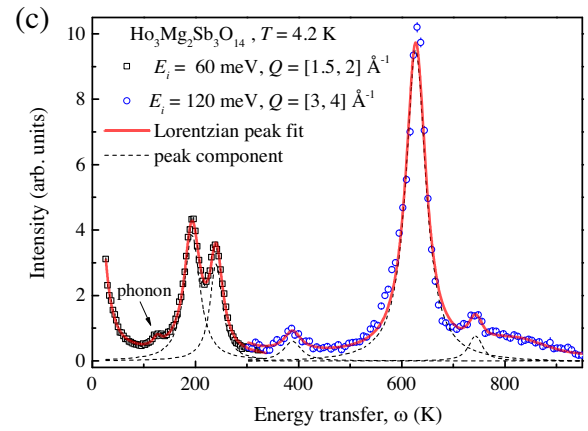

(c)

(f)

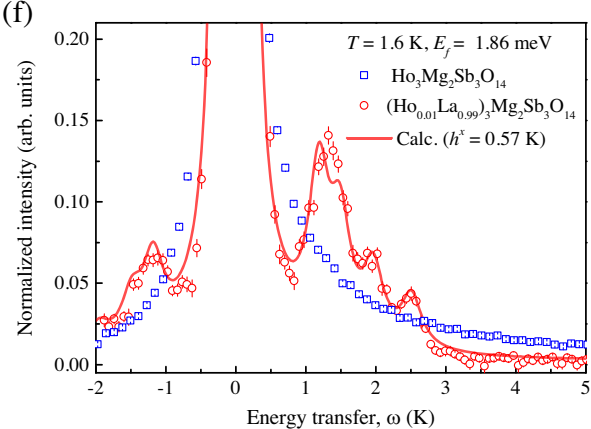

FIG. 2. (a) Simplified partial crystal structure of $\mathrm{Ho}_{3} \mathrm{Mg}_{2} \mathrm{Sb}_{3} \mathrm{O}_{14}$, showing alternating $\mathrm{Ho}^{3+}$ kagome layers (large blue spheres) and $\mathrm{Mg}^{2+}$ triangular layers (small orange spheres). (b) Local environment of $\mathrm{Ho}^{3+}$ ions, showing eight oxygen ions (small pink spheres), two $\mathrm{Mg}^{2+}$ ions, and four nearest-neighbor $\mathrm{Ho}^{3+}$ ions around a central $\mathrm{Ho}^{3+}$ ion. The tripodlike arrangement of the local Ising axes is enforced by the oxygen in the center of each $\mathrm{MgHo}_{3}$, which yields a canting angle of $22.3^{\circ}$ with respect to the kagome plane. Spins are labeled by black arrows while $Q_{j}=+1$ and $Q_{j}=-1$ magnetic charges are illustrated by red and blue spheres, respectively. The coordinate system depicts the local Ising, transverse field, and mean-field directions. (c) Crystal-field excitations measured by inelastic neutron-scattering experiments on the SEQUOIA spectrometer. Open black circles and blue squares indicate intensities measured with incident neutron energies of 60 and $120 \mathrm{meV}$, respectively. Five crystal-field levels can be directly resolved at energies of 190(2), 238(2), 388(9), 627(5), and 743(9) K, whose peak positions are extracted from Lorentzian fits to the data (black dashed lines). The overall fit, including a modeled phonon background, is shown as a red line. Detailed analysis of the crystal-field excitations can be found in a separate study [53]. (d) Comparison of the single-ion response expected for a $\mathrm{Ho}^{3+}$ ion in a pyrochlore versus a tripod-kagome geometry. Crystal-field singlets $|0\rangle$ and $|1\rangle$ in $\mathrm{Ho}_{3} \mathrm{Mg}_{2} \mathrm{Sb}_{3} \mathrm{O}_{14}$ can be effectively described as a transverse field $h^{x}$ acting on the nonKramers doublet $| \pm\rangle$ of $\mathrm{Ho}_{2} \mathrm{Ti}_{2} \mathrm{O}_{7}$. While hyperfine interactions split the electronic-nuclear manifold into eight levels in both systems, the transverse field $h^{x}$ transforms the classical single-ion picture of $\mathrm{Ho}_{2} \mathrm{Ti}_{2} \mathrm{O}_{7}$ into a quantum picture in $\mathrm{Ho}_{3} \mathrm{Mg}_{2} \mathrm{Sb}_{3} \mathrm{O}_{14}$, where each eigenstate is a quantum superposition of $\left|+, I^{z}\right\rangle$ and $\left|-, I^{z}\right\rangle$. Red dashed arrows mark the allowed transitions between eigenstates of the same $I^{z}$. (e) Calculated scattering intensity of single-ion excitations as a function of $h^{x}$ at a temperature of $1.6 \mathrm{~K}$. Dashed lines present the transition energies whose intensities are calculated and convoluted with a Lorentzian function with FWHM $=0.35 \mathrm{~K}$. (f) Low-energy inelastic neutron-scattering response measured on the OSIRIS spectrometer for pure $\mathrm{Ho}_{3} \mathrm{Mg}_{2} \mathrm{Sb}_{3} \mathrm{O}_{14}$ (SG sample) and the dilute Hotripod magnet $\left(\mathrm{Ho}_{0.01} \mathrm{La}_{0.99}\right)_{3} \mathrm{Mg}_{2} \mathrm{Sb}_{3} \mathrm{O}_{14}$ at a temperature of $1.6 \mathrm{~K}$. The two datasets are normalized to the same total spectrum weight. Solid lines represent the single-ion spectrum calculated using $h^{x}=0.57 \mathrm{~K}$, which yields the best agreement with the experimental data.

increase quantum fluctuations with fine-tuning. Other features, such as structural disorder or couplings to other degrees of freedom, usually suppress coherent quantum tunneling. Uncovering such effects and elucidating their role is a crucial and necessary step toward the experimental realization and engineering of genuinely quantum phases, such as the putative QSF state mentioned above.

In this work, we present comprehensive inelastic neutron scattering data which uncover spin dynamics at the lowest measurable temperatures $(\approx 0.1 \mathrm{~K})$ in the dipolar kagome Ising magnet $\mathrm{Ho}_{3} \mathrm{Mg}_{2} \mathrm{Sb}_{3} \mathrm{O}_{14}$ [50]. This material is one of a series of "tripod-kagome" materials derived from the pyrochlore structure by chemical substitution, yielding kagome planes of magnetic rare-earth ions separated by triangular planes of nonmagnetic $\mathrm{Mg}^{2+}$ ions [Fig. 2(a)]. Previous measurements of isostructural $\mathrm{Dy}_{3} \mathrm{Mg}_{2} \mathrm{Sb}_{3} \mathrm{O}_{14}$ revealed a CSF state at low temperature [41], in which no spin dynamics were observed in either neutronscattering or ac-susceptibility data [41,51]. Our measurements on $\mathrm{Ho}_{3} \mathrm{Mg}_{2} \mathrm{Sb}_{3} \mathrm{O}_{14}$ uncover a spin-fragmented state with instantaneous magnetic correlations closely resembling those of $\mathrm{Dy}_{3} \mathrm{Mg}_{2} \mathrm{Sb}_{3} \mathrm{O}_{14}$. Yet, we observe structured dynamic magnetic correlations at low temperature, indicating persistent spin dynamics in sharp contrast to the $\mathrm{Dy}^{3+}$ compound. We show that this stems from the low symmetry of the tripod-kagome structure and the non-Kramers nature 
of the $\mathrm{Ho}^{3+}$ ion, the combination of which generates an effective local magnetic field transverse to the Ising magnetic dipole moments. The effective low-energy Hamiltonian for $\mathrm{Ho}_{3} \mathrm{Mg}_{2} \mathrm{Sb}_{3} \mathrm{O}_{14}$ thus maps onto an iconic model of quantum magnetism-interacting Ising spins in a transverse magnetic field [52]. We use neutron-scattering experiments to determine this Hamiltonian, and employ a combination of exact diagonalization (ED), field theoretic, and Monte Carlo (MC) methods to understand its spin correlations and excitations. Our calculations complement previous studies of the quantum kagome ice model $[11,35,36,46,48]$ by including dipolar interactions to delineate the putative role played by transverse fields to stabilize partially disordered quantum phases. In $\mathrm{Ho}_{3} \mathrm{Mg}_{2} \mathrm{Sb}_{3} \mathrm{O}_{14}$, however, the physics of $\mathrm{Ho}^{3+}$ ions is profoundly affected by the strong nuclear hyperfine coupling, which eventually destroys coherent quantum effects between sites. As a result, $\mathrm{Ho}_{3} \mathrm{Mg}_{2} \mathrm{Sb}_{3} \mathrm{O}_{14}$ realizes a spin-fragmented state with predominantly single-ion quantum fluctuations. A key insight of our work is thus to determine the interplay of two effects-intrinsic transverse field and hyperfine coupling — on the quantum dynamics of a highly frustrated magnet.

The paper is structured as follows. In Sec. II, we summarize the experimental methods that we employ. In Sec. III, we present neutron-scattering measurements of the crystal-field excitations of $\mathrm{Ho}_{3} \mathrm{Mg}_{2} \mathrm{Sb}_{3} \mathrm{O}_{14}$ and of a structurally analogous but magnetically dilute system $\left(\mathrm{Ho}_{0.01} \mathrm{La}_{0.99}\right)_{3} \mathrm{Mg}_{2} \mathrm{Sb}_{3} \mathrm{O}_{14}$ and use these measurements to parametrize the spin Hamiltonian of $\mathrm{Ho}_{3} \mathrm{Mg}_{2} \mathrm{Sb}_{3} \mathrm{O}_{14}$. In $\mathrm{Sec}$. IV, we report heat-capacity measurements that identify a magnetic phase transition at $T^{*}=0.32 \mathrm{~K}$ accompanied by a large specific-heat feature of coupled electronicnuclear origin. In Sec. V, we report low-temperature inelastic neutron-scattering measurements on polycrystalline samples of $\mathrm{Ho}_{3} \mathrm{Mg}_{2} \mathrm{Sb}_{3} \mathrm{O}_{14}$. They reveal that spin fragmentation occurs below $T^{*}$, and that low-energy spin excitations are structured in both momentum and energy space at the lowest measurable temperatures. In Sec. VI, we use theoretical modeling to understand our data. Finally, we conclude in Sec. VII with a discussion of the general implications of our study.

\section{METHODS}

Two different polycrystalline samples of $\mathrm{Ho}_{3} \mathrm{Mg}_{2} \mathrm{Sb}_{3} \mathrm{O}_{14}$ were prepared for this study, the first using a traditional solid state reaction method (referred to as SS sample), and the second using a sol-gel method (referred to as SG sample). For the $\mathrm{SS}$ sample, stoichiometric ratios of $\mathrm{Ho}_{2} \mathrm{O}_{3}$ (99.9\%), $\mathrm{MgO}$ (99.99\%), and $\mathrm{Sb}_{2} \mathrm{O}_{3}$ (99.99\%) fine powder were carefully ground and reacted at a temperature of $1350^{\circ} \mathrm{C}$ in air for $24 \mathrm{~h}$. This heating step was repeated until the amount of impurity phases as determined by $\mathrm{x}$-ray diffraction was not reduced further. The synthesized SS sample contained a small amount of $\mathrm{Ho}_{3} \mathrm{SbO}_{7}$ impurity [2.29(18)wt \%], which orders antiferromagnetically at $T_{N}=2.07 \mathrm{~K}$ [54]. This impurity can be removed by the sol-gel synthesis method. For this synthesis, stoichiometric amounts of $\mathrm{Ho}\left(\mathrm{NO}_{3}\right)_{3}, \mathrm{Mg}\left(\mathrm{NO}_{3}\right)_{3}$ (prepared by dissolving $\mathrm{Ho}_{2} \mathrm{O}_{3}$ and $\mathrm{MgO}$ in hot diluted nitric acid solution) and antimony tartarate (prepared by dissolving $\mathrm{Sb}_{2} \mathrm{O}_{3}$ in hot tartaric acid solution) were first mixed in a beaker. Citrate acid with a metal-to-citrate molar ratio of $1: 2$ was then added to the solution followed by a subsequent heating on a hot plate at $120^{\circ} \mathrm{C}$ overnight to remove excessive water. The obtained gel-like solution was slowly heated to $200{ }^{\circ} \mathrm{C}$ in a box furnace to decompose the nitrate, and was pyrolyzed at $600{ }^{\circ} \mathrm{C}$ for $10-12 \mathrm{~h}$ in air. The obtained powder was then ground, pressed into a pellet, and reheated at $1300^{\circ} \mathrm{C}$ until a well-reacted crystalline powder was obtained. Heat-capacity measurements presented below show that the thermomagnetic behavior of the two samples is almost identical, except for a small peak around $2.1 \mathrm{~K}$ in the SS sample originating from the $\mathrm{Ho}_{3} \mathrm{SbO}_{7}$ impurity. A Ho-diluted (La-doped) sample of $\left(\mathrm{Ho}_{0.01} \mathrm{La}_{0.99}\right)_{3} \mathrm{Mg}_{2} \mathrm{Sb}_{3} \mathrm{O}_{14}$ was also synthesized with the same sol-gel technique, with $99 \% \quad \mathrm{Ho}_{2} \mathrm{O}_{3}$ replaced by $\mathrm{La}_{2} \mathrm{O}_{3}$ powder $\left(99.9 \%\right.$, baked at $900{ }^{\circ} \mathrm{C}$ overnight before use).

Low-temperature specific-heat measurements were performed on a Quantum Design Physical Properties Measurement System instrument using dilution refrigerator $(0.07 \leq T \leq 4 \mathrm{~K})$ and standard $(1.6 \leq T \leq 100 \mathrm{~K})$ probes. For the dilution refrigerator measurement, the powder samples were cold sintered with Ag powder. The contribution of the Ag powder was measured separately and subtracted from the data. The lattice contribution to the heat capacity was estimated from measurements of the isostructural nonmagnetic compound $\mathrm{La}_{3} \mathrm{Mg}_{2} \mathrm{Sb}_{3} \mathrm{O}_{14}$.

Powder X-ray diffraction measurements were carried out with $\mathrm{Cu} K \alpha$ radiation $(\lambda=1.5418 \AA)$ in transmission mode. Powder neutron-diffraction measurements were carried out using the HB-2A high-resolution powder diffractometer [55] at the High Flux Isotope Reactor at Oak Ridge National Laboratory, with a neutron wavelength of $1.546 \AA$. Rietveld refinements of the crystal and magnetic structures were carried out using the FULLPROF suite of programs [56]. Peak shapes were modeled by Thompson-Cox-Hastings pseudo-Voigt functions, and backgrounds were fitted using Chebyshev polynomial functions.

Inelastic neutron-scattering measurements on the SS sample of $\mathrm{Ho}_{3} \mathrm{Mg}_{2} \mathrm{Sb}_{3} \mathrm{O}_{14}$ were carried out using the Fine-Resolution Fermi Chopper Spectrometer (SEQUOIA) [57] at the Spallation Neutron Source of Oak Ridge National Laboratory and the Disk Chopper Spectrometer (DCS) [58] at the NIST Center for Neutron Research. For the SEQUOIA experiment, a $\sim 5$ g powder sample of $\mathrm{Ho}_{3} \mathrm{Mg}_{2} \mathrm{Sb}_{3} \mathrm{O}_{14}$ was loaded in an aluminum sample container and cooled to $4 \mathrm{~K}$ with a closed-cycle refrigerator. Data were measured with incident neutron energies of 120,60 , and $8 \mathrm{meV}$. The same 
measurements were repeated for an empty aluminum sample holder and used for background subtraction. For the DCS measurements, the same sample was loaded in a copper can, filled with 10 bar of helium gas at room temperature, and cooled to millikelvin temperatures using a dilution refrigerator. The measurements were carried out with an incident neutron energy of $3.27 \mathrm{meV}$ at temperatures between 0.12 and $40 \mathrm{~K}$. Measurements of an empty copper sample holder were also made and used for background subtractions. Because of the large specific heat and related relaxation processes below $1 \mathrm{~K}$, a thermal stabilization time of $6 \mathrm{~h}$ was used; no change in the data was observed after this waiting time. Data reduction was performed using the DAVE program [59]. For modeling and fitting purposes, data were corrected for background scattering using empty-container measurements and/or high-temperature measurements, as specified in the text. These data were also corrected for neutron absorption [60], placed on an absolute intensity scale by scaling to the nuclear Bragg profile, and the magnetic scattering from the $\mathrm{Ho}_{3} \mathrm{SbO}_{7}$ impurity below its $T_{N}$ of $2.07 \mathrm{~K}$ was subtracted as described in Ref. [41]. Additional inelastic neutronscattering measurements on $\mathrm{Ho}_{3} \mathrm{Mg}_{2} \mathrm{Sb}_{3} \mathrm{O}_{14}$ (SG sample) and $\left(\mathrm{Ho}_{0.01} \mathrm{La}_{0.99}\right)_{3} \mathrm{Mg}_{2} \mathrm{Sb}_{3} \mathrm{O}_{14}$ were carried out using the OSIRIS backscattering spectrometer at the ISIS neutron source with a final neutron energy of $1.84 \mathrm{meV}$. For $\left(\mathrm{Ho}_{0.01} \mathrm{La}_{0.99}\right)_{3} \mathrm{Mg}_{2} \mathrm{Sb}_{3} \mathrm{O}_{14}$, a $13.6 \mathrm{~g}$ powder sample was loaded into an aluminum can and was cooled with an Orange cryostat to the base temperature of $1.6 \mathrm{~K}$. For $\mathrm{Ho}_{3} \mathrm{Mg}_{2} \mathrm{Sb}_{3} \mathrm{O}_{14}$, $4 \mathrm{~g}$ of SG powder was wrapped in a thin copper foil and placed into a copper sample can with a capillary that allowed helium filling at low temperature. The system was cooled using a dilution refrigerator, and with a maximum of 3 bar filled helium, the lowest sample temperature accessed in this experiment was estimated to be $400 \pm 50 \mathrm{mK}$ by comparison with the DCS data.

For convenience, in the following sections, we use a unit system with $k_{B}=1$ and $\hbar=1$, so that all energies are given in units of $\mathrm{K}$.

\section{EFFECTIVE HAMILTONIAN}

\section{A. Crystal structure and interactions}

The crystal structure of $\mathrm{Ho}_{3} \mathrm{Mg}_{2} \mathrm{Sb}_{3} \mathrm{O}_{14}$ (space group $R \overline{3} \mathrm{~m}$ ) is shown in Fig. 2(a) and contains kagome planes of magnetic $\mathrm{Ho}^{3+}$ ions separated by triangular layers of nonmagnetic $\mathrm{Mg}^{2+}$ [50]. The $\mathrm{Ho}^{3+}$ site has $C_{2 h}$ point symmetry and its local environment contains eight oxygen atoms [50,51]. The orientations of $\mathrm{Ho}^{3+}$ magnetic dipole moments are constrained by crystal electric field effects to point along the line connecting $\mathrm{Ho}^{3+}$ to its two closest oxygen neighbors, which are situated near the centroids of the $\mathrm{MgHo}_{3}$ tetrahedra [Fig. 2(b)]. Rietveld corefinements to X-ray and neutron powder-diffraction data for $\mathrm{Ho}_{3} \mathrm{Mg}_{2} \mathrm{Sb}_{3} \mathrm{O}_{14}$ (SS sample) confirm this crystal structure, and reveal a small amount of $\mathrm{Ho}^{3+} / \mathrm{Mg}^{2+}$ site mixing such that $3.2(2) \%$ of $\mathrm{Ho}^{3+}$ atomic positions are occupied in a disordered way by $\mathrm{Mg}^{2+}$ (see the Appendix A). Hence, the extent of chemical disorder in $\mathrm{Ho}_{3} \mathrm{Mg}_{2} \mathrm{Sb}_{3} \mathrm{O}_{14}$ is less than in its $\mathrm{Dy}^{3+}$ analog, where the corresponding value is 6(2)\% [41].

We anticipate that the spin Hamiltonian for $\mathrm{Ho}_{3} \mathrm{Mg}_{2} \mathrm{Sb}_{3} \mathrm{O}_{14}$ may be written as a sum of three terms,

$$
\mathcal{H}=\mathcal{H}_{\mathrm{cf}}+\mathcal{H}_{\text {hf }}+\mathcal{H}_{\text {int }},
$$

where $\mathcal{H}_{\mathrm{cf}}, \mathcal{H}_{\mathrm{hf}}$, and $\mathcal{H}_{\text {int }}$ denote, respectively, the crystal-field, nuclear hyperfine, and pairwise interaction Hamiltonian. We now consider the origin, form, and magnitude of each term, and show that they are all relevant in $\mathrm{Ho}_{3} \mathrm{Mg}_{2} \mathrm{Sb}_{3} \mathrm{O}_{14}$ at low temperature.

\section{B. Crystal-field Hamiltonian}

We use inelastic neutron-scattering measurements and point-charge calculations to determine the parameters of $\mathcal{H}_{\mathrm{cf}}$. The high-energy spectrum observed in $\mathrm{Ho}_{3} \mathrm{Mg}_{2} \mathrm{Sb}_{3} \mathrm{O}_{14}$ (SS sample) comprises five crystal-field excitations, with energies and relative intensities that resemble those of pyrochlore spin ice $\mathrm{Ho}_{2} \mathrm{Ti}_{2} \mathrm{O}_{7}$ [61,62] except for an overall downward renormalization in energy [Fig. 2(c)]. This overall resemblance is expected given the similar local environments for $\mathrm{Ho}^{3+}$ ions in these two systems. However, a crucial difference stems from the reduced $C_{2 h}$ symmetry of the $\mathrm{Ho}^{3+}$ site in $\mathrm{Ho}_{3} \mathrm{Mg}_{2} \mathrm{Sb}_{3} \mathrm{O}_{14}$ compared to the $D_{3 d}$ symmetry in $\mathrm{Ho}_{2} \mathrm{Ti}_{2} \mathrm{O}_{7}$. Whereas the crystal-field ground state in $\mathrm{Ho}_{2} \mathrm{Ti}_{2} \mathrm{O}_{7}$ is a non-Kramers doublet, in $\mathrm{Ho}_{3} \mathrm{Mg}_{2} \mathrm{Sb}_{3} \mathrm{O}_{14}$ all crystal-field levels are necessarily singlets [50]. However, as a probable consequence of spin-spin interactions (see Sec. V), the excitation associated with the splitting of the ground-state doublet is strongly overdamped [Fig. 2(f)]. This precludes a direct neutron-scattering measurement of this energy splitting in $\mathrm{Ho}_{3} \mathrm{Mg}_{2} \mathrm{Sb}_{3} \mathrm{O}_{14}$. We addressed this problem using two complementary approaches. First, we performed pointcharge calculations using an effective charge model that matches the high-energy crystal-field excitation spectrum (see Appendix B) [53]. Second, we validated the lowenergy predictions of this model using high-resolution neutron scattering measurements on a magnetically dilute Ho tripod-kagome compound, $\left(\mathrm{Ho}_{0.01} \mathrm{La}_{0.99}\right)_{3} \mathrm{Mg}_{2} \mathrm{Sb}_{3} \mathrm{O}_{14}$, such that interaction effects between sites are negligible. We discuss these results in turn below.

Our point-charge model [53] predicts that the groundstate doublet is split into two singlets separated by an energy gap $\Delta \approx 1.74 \mathrm{~K}$ (see Appendix B). The two singlets are well approximated by symmetric and antisymmetric superpositions of pure free-ion states,

$$
\begin{aligned}
& |0\rangle \approx \frac{1}{\sqrt{2}}(|+\rangle+|-\rangle), \\
& |1\rangle \approx \frac{1}{\sqrt{2}}(|+\rangle-|-\rangle),
\end{aligned}
$$


where $| \pm\rangle \equiv\left|J=8, J^{z}= \pm 8\right\rangle$ represents the ground-state doublet of $\mathrm{Ho}^{3+}$ in $\mathrm{Ho}_{2} \mathrm{Ti}_{2} \mathrm{O}_{7}$ if we ignore the small contributions from other $J^{z}$ components $[61,63]$. At low temperatures, only $|0\rangle$ and $|1\rangle$ are thermally populated because of their $190 \mathrm{~K}$ separation from higher-energy crystal-field levels [Fig. 2(c)], which is consistent with higher-temperature inelastic neutron data [53] and specificheat measurements (Sec. IV). The form of $|0\rangle$ and $|1\rangle$ allows for a nonzero angular momentum matrix element $\left\langle 0\left|\hat{J}^{z}\right| 1\right\rangle \approx 8$, while $\left\langle 0\left|\hat{J}^{\alpha}\right| 1\right\rangle$ with $\alpha=x, y$ are vanishingly small (more accurate values are given in Appendix B). Similar to the procedure for pyrochlore spin ices [63], we construct Pauli matrices using the two thermally accessible crystal-field states,

$$
\sigma^{ \pm}=2| \pm\rangle\left\langle\mp\left|, \quad \sigma^{z}=\right|+\right\rangle\langle+|-|-\rangle\langle-| .
$$

In this framework, the total magnetic dipole moment operator is related to $\sigma^{z}$ as

$$
\boldsymbol{\mu}=-g_{J} \mu_{B} \sigma^{z}\left\langle 0\left|\hat{J}^{z}\right| 1\right\rangle \hat{\mathbf{z}},
$$

where $g_{J}=\frac{5}{4}$ is the $\mathrm{Ho}^{3+}$ Landé factor and $\hat{\mathbf{z}}$ is a local Ising axis shown in Fig. 2(b). The nonzero matrix element $\left\langle 0\left|\hat{J}^{z}\right| 1\right\rangle$ is therefore expected to generate a total magnetic moment of magnitude $9.74 \mu_{B}$, which is conserved. In contrast, static moments only appear when $\left\langle\sigma^{z}\right\rangle$ becomes nonzero, e.g., under an external magnetic field. Meanwhile, $\sigma^{x}$ and $\sigma^{y}$ transform as higher-order multipoles which are not directly observable in our neutron-scattering measurements. These predictions are supported by our isothermal magnetization measurements between 1.8 and $40 \mathrm{~K}$, which are consistent with a model of paramagnetic Ising spins (see Appendix C).

It is established [14,52] that an energy splitting between two crystal-field singlets can be exactly mapped into a transverse magnetic field acting on a corresponding doublet. This mapping can be understood by recognizing that $|0\rangle$ and $|1\rangle$ are the eigenstates of the

$$
\sigma^{x}=\left(\begin{array}{ll}
0 & 1 \\
1 & 0
\end{array}\right)
$$

Pauli matrix. Therefore, in the Pauli matrix picture, our crystal-field Hamiltonian can be recast as

$$
\mathcal{H}_{\mathrm{cf}}=h^{x} \sigma^{x}
$$

where $h^{x}=\Delta / 2$ is the intrinsic transverse field [Fig. 2(d)]. We will use this pseudospin representation in the rest of this paper.

\section{Nuclear hyperfine Hamiltonian}

Hyperfine interactions couple nonzero nuclear spins to the local magnetic field from surrounding electrons. With a nuclear spin quantum number $I=7 / 2,{ }^{165} \mathrm{Ho}$ is the only stable isotope of holmium and its hyperfine energy scale is the strongest among the rare-earth elements. For a nonKramers electronic system in the pseudospin approximation, the hyperfine Hamiltonian takes the simple form [64],

$$
\mathcal{H}_{\mathrm{hf}}=A_{\mathrm{hf}} I^{z} \sigma^{z}
$$

where $I^{z}=-I, \ldots, I$ labels the $z$ component of the nuclear spin operator and $A_{\mathrm{hf}}=0.319 \mathrm{~K}$ is the hyperfine coupling constant for Ho $[65,66]$. We neglect the electric quadrupole coupling constant because its energy scale $(P=0.004 \mathrm{~K})$ is very small.

In $\mathrm{Ho}_{2} \mathrm{Ti}_{2} \mathrm{O}_{7}$, the hyperfine coupling splits the combined electronic and nuclear system into eight uniformly spaced levels; each level is doubly degenerate because of the Kramers degeneracy of the combined electronic and nuclear spin system [Fig. 2(d)]. At the single-ion level, the system remains classical because both $I^{z}$ and $\sigma^{z}$ are good quantum numbers, and eigenstates can be labeled as $\left| \pm, I^{z}\right\rangle$. By contrast, for $\mathrm{Ho}_{3} \mathrm{Mg}_{2} \mathrm{Sb}_{3} \mathrm{O}_{14}$, the single-ion Hamiltonian contains both an intrinsic transverse field and hyperfine interactions,

$$
\mathcal{H}_{\mathrm{SI}}=\mathcal{H}_{\mathrm{cf}}+\mathcal{H}_{\mathrm{hf}}
$$

This generates a dynamic electronic moment since neither $\sigma^{z}$ nor $\sigma^{x}$ are good quantum numbers. Diagonalizing the 16-dimensional $\mathcal{H}_{\mathrm{SI}}$ yields an unevenly spaced spectrum for which each eigenstate is a quantum superposition of $\left|+, I^{z}\right\rangle$ and $\left|-, I^{z}\right\rangle$ whose mixing depends on the values of $I^{z}$ and $h^{x}$, and the lowest energy states always have $I^{z}= \pm 7 / 2$. To illustrate this effect, Fig. 2(e) shows the low-temperature magnetic scattering intensity of electronic spins as a function of $h^{x}$. For vanishing $h^{x}$, the magnetic response is purely elastic because the dipolar matrix elements connecting different eigenstates are zero. With increasing $h^{x}$, the scattering acquires an inelastic component that comprises four excitations, arising from transitions between eigenstates with the same $I^{z}$ [Fig. 2(d)]. Finally, in the limit of $h^{x} \gg A_{\mathrm{hf}} I$, a single crystal-field excitation at $\Delta=2 h^{x}$ is obtained.

To test our model single-ion Hamiltonian against experiment, we employ high-resolution neutron scattering measurements of the magnetically dilute Ho tripod-kagome compound $\left(\mathrm{Ho}_{0.01} \mathrm{La}_{0.99}\right)_{3} \mathrm{Mg}_{2} \mathrm{Sb}_{3} \mathrm{O}_{14}$. We assume that the $\mathrm{Ho}^{3+}$ ions are randomly distributed on the kagome lattice and pairwise interactions between them are negligible. Neutron-scattering data measured at $1.6 \mathrm{~K}$ are shown in Fig. 2(f); they display a broad and intense peak at $\omega=1.3 \mathrm{~K}$, along with two narrower and weaker side peaks at $\omega=1.9$ and $2.5 \mathrm{~K}$. The experimental data are 
in excellent quantitative agreement with exact diagonalization calculations of $H_{\mathrm{SI}}$, taking $h^{x}=0.57 \mathrm{~K}$ [Fig. 2(e)]. This result yields strong evidence that $H_{\mathrm{SI}}$ describes well the single-ion properties of Ho-based tripod-kagome magnets. Importantly, due to the larger ionic size of $\mathrm{La}^{3+}$ compared with $\mathrm{Ho}^{3+}$, the lattice parameters of $\left(\mathrm{Ho}_{0.01} \mathrm{La}_{0.99}\right)_{3} \mathrm{Mg}_{2} \mathrm{Sb}_{3} \mathrm{O}_{14}$ are approximately 3\% larger than those of $\mathrm{Ho}_{3} \mathrm{Mg}_{2} \mathrm{Sb}_{3} \mathrm{O}_{14}$. Using a power-law scaling of $h^{x}$ as a function of lattice parameters, we extrapolate to $h^{x}=0.85 \mathrm{~K}$ in $\mathrm{Ho}_{3} \mathrm{Mg}_{2} \mathrm{Sb}_{3} \mathrm{O}_{14}$ [53], which is in good agreement with the point-charge estimate of $h^{x}=0.77 \mathrm{~K}$. We take $h^{x}=0.85 \mathrm{~K}$ for $\mathrm{Ho}_{3} \mathrm{Mg}_{2} \mathrm{Sb}_{3} \mathrm{O}_{14}$ throughout the rest of this paper.

\section{Transverse Ising model}

We now consider the effect of pairwise interactions $J_{i j}$ between $\mathrm{Ho}^{3+}$ ions. By analogy with spin-ice pyrochlores [3] and isostructural $\mathrm{Dy}_{3} \mathrm{Mg}_{2} \mathrm{Sb}_{3} \mathrm{O}_{14}$ [41], we expect that $J_{i j}$ contains a combination of nearest-neighbor exchange interactions $J_{\mathrm{NN}}$ and long-range magnetic dipolar interactions of overall scale $D$. In principle, interactions between transverse spin components are also possible, but they are expected to be several orders of magnitude smaller [63], so we do not consider them further. The pairwise interaction Hamiltonian is therefore

$$
\mathcal{H}_{\text {int }}=\frac{1}{2} \sum_{i, j} J_{i j} \sigma_{i}^{z} \sigma_{j}^{z}
$$

with

$J_{i j}=J_{\mathrm{NN}} \delta_{r_{i j}, r_{\mathrm{NN}}}+D r_{\mathrm{NN}}^{3} \frac{\hat{\mathbf{z}}_{i} \cdot \hat{\mathbf{z}}_{j}-3\left(\hat{\mathbf{z}}_{i} \cdot \hat{\mathbf{r}}_{i j}\right)\left(\hat{\mathbf{z}}_{j} \cdot \hat{\mathbf{r}}_{i j}\right)}{r_{i j}^{3}}$,

where $\delta_{r_{i j}, r_{\mathrm{NN}}}$ is the Kronecker delta function, $r_{\mathrm{NN}}$ is the distance between nearest-neighbor $\mathrm{Ho}^{3+}$ ions, $r_{i j}$ is the distance between ions at positions $\mathbf{r}_{i}$ and $\mathbf{r}_{j}$, and $\hat{\mathbf{r}}_{i j}=$ $\left(\mathbf{r}_{i}-\mathbf{r}_{j}\right) / r_{i j}$. The value of $D=\mu_{0} \mu^{2} /\left(4 \pi k_{B} r_{\mathrm{NN}}^{3}\right)=1.29 \mathrm{~K}$ is fixed by the crystal structure, and we will obtain an experimental estimate of $J_{\mathrm{NN}} \approx-0.64 \mathrm{~K}$ in Sec. V. With $\hat{\mathbf{z}}_{i} \cdot \hat{\mathbf{z}}_{j}=-0.28$, and $-3\left(\hat{\mathbf{z}}_{i} \cdot \hat{\mathbf{r}}_{\mathrm{NN}}\right)\left(\hat{\mathbf{z}}_{j} \cdot \hat{\mathbf{r}}_{\mathrm{NN}}\right)=1.93$, the sum of exchange and dipolar couplings at the nearest-neighbor level is approximately $1.69 \mathrm{~K}$, and hence antiferromagnetic in the pseudospin language. Consequently, magnetic interactions between sites are frustrated and, furthermore, comparable in magnitude to $h^{x}$.

Using the results of the previous sections, we rewrite the full spin Hamiltonian, Eq. (1), as

$$
\mathcal{H}=A_{\mathrm{hf}} \sum_{i} I_{i}^{z} \sigma_{i}^{z}+h^{x} \sum_{i} \sigma_{i}^{x}+\frac{1}{2} \sum_{i, j} J_{i j} \sigma_{i}^{z} \sigma_{i}^{z} .
$$

Equation (10) is equivalent in form to an Ising model in a transverse field (TIM), with an additional on-site longitudinal field due to the hyperfine coupling. The TIM has been used to model diverse physical phenomena, including ferroelectricity [67,68], superconductivity [69], quantum information [70,71], and quantum phase transitions [72,73]. Typically, the pairwise interactions that drive magnetic ordering compete with the transverse field that drives quantum tunneling. In the absence of geometrical frustration, a phase transition only occurs to a magnetically ordered state if $J_{i j}$ dominates over $h^{x}$. The interplay of frustration and transverse field may generate exotic quantum phases $[11,14,46,48]$. On the kagome lattice, the TIM with nearest-neighbor antiferromagnetic interactions is predicted to have a quantum-disordered ground state for small $h^{x}$, smoothly connected to a quantum paramagnetic state at large $h^{x}[11,46,48]$. On the pyrochlore lattice, an external field cannot be applied transverse to all spins simultaneously because the different local Ising axes are not coplanar, and a homogeneous transverse field that emerges at single-ion level is absent in chemically ordered pyrochlores. However, transverse fields generated by spinspin interactions are related to monopole hopping in spin ice [74], and transverse fields generated by chemical disorder have been identified as a possible route to pyrochlore quantum spin liquid states [14], and used to explain the spin dynamics of $\mathrm{Pr}_{2} \mathrm{Zr}_{2} \mathrm{O}_{7}$ [22,24] and $\mathrm{Tb}_{2} \mathrm{Ti}_{2} \mathrm{O}_{7}[75,76]$. Nevertheless, a potential challenge to modeling such materials is that chemical disorder generates a broad distribution of transverse fields in the sample [77]. Hence, a key feature of $\mathrm{Ho}_{3} \mathrm{Mg}_{2} \mathrm{Sb}_{3} \mathrm{O}_{14}$ is that its transverse field is intrinsic to the chemically ordered structure and is homogeneous to a first approximation.

\section{SPECIFIC-HEAT MEASUREMENTS}

We use heat-capacity measurements to understand thermodynamic properties of $\mathrm{Ho}_{3} \mathrm{Mg}_{2} \mathrm{Sb}_{3} \mathrm{O}_{14}$ and identify possible phase transitions. A sharp peak in the magnetic specific heat $\left(C_{m}\right)$ is observed at $T^{*}=0.32 \mathrm{~K}$ for both SS and SG samples, indicating a symmetry-breaking magnetic phase transition [Fig. 3(a)]. The value of $T^{*}$ is consistent with the broad peak previously observed around $0.4 \mathrm{~K}$ using the ac-susceptibility technique [50]. Whereas the ac-susceptibility peak is frequency dependent [50], the sharpness of the $C_{m}$ peak is inconsistent with a conventional spin freezing scenario. The value of $T^{*}$ is also close to the temperature at which the isostructural compound $\mathrm{Dy}_{3} \mathrm{Mg}_{2} \mathrm{Sb}_{3} \mathrm{O}_{14}$ undergoes a phase transition from a kagome spin-ice state to a CSF state $(\sim 0.3 \mathrm{~K}$ in Ref. [41] and $\sim 0.37 \mathrm{~K}$ in Ref. [51]). As we show in Sec. V, $T^{*}$ corresponds to the onset of a spin-fragmented state in $\mathrm{Ho}_{3} \mathrm{Mg}_{2} \mathrm{Sb}_{3} \mathrm{O}_{14}$, characterized by a reduced ordered moment compared to a CSF state.

Below $1 \mathrm{~K}$, a broad specific-heat feature is observed in addition to the sharp peak, consistent with a nuclear spin contribution. By integrating $C_{m} / T$ from $20 \mathrm{~K}$ to the lowest measurement temperature of $76 \mathrm{mK}$, the recovered 


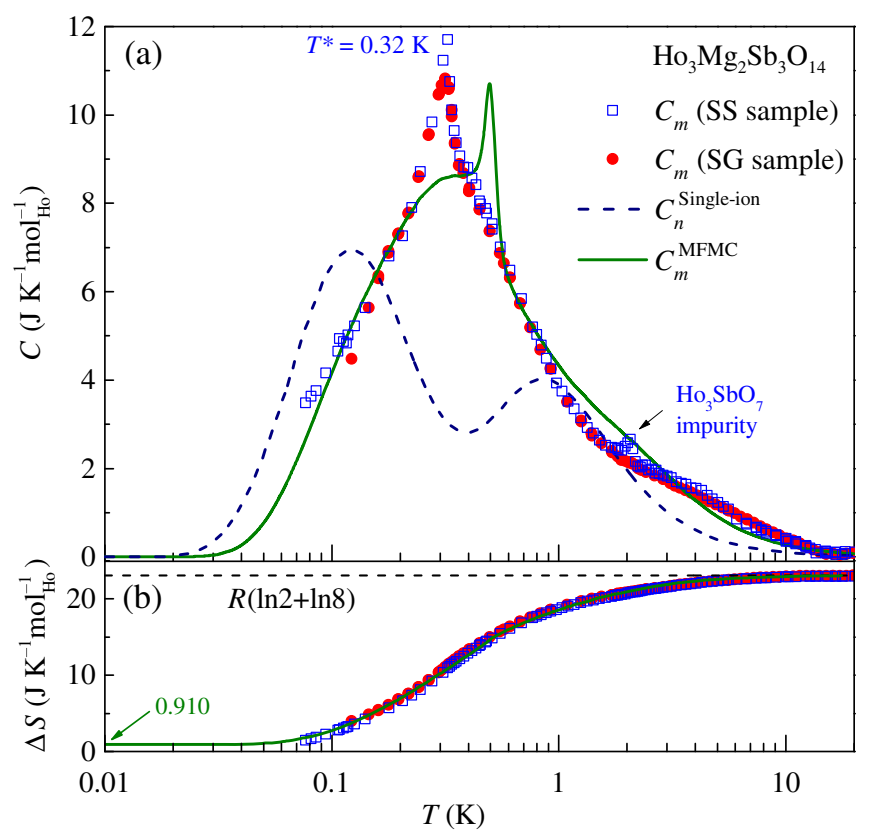

FIG. 3. (a) Magnetic contribution to the specific heat in $\mathrm{Ho}_{3} \mathrm{Mg}_{2} \mathrm{Sb}_{3} \mathrm{O}_{14}\left(C_{m}\right.$, shown as blue squares for the SS sample and red dots for the SG sample). The specific heat contains coupled electronic and nuclear spin contributions. The navy dashed line shows the single-ion nuclear specific heat calculated assuming an isolated $\mathrm{Ho}^{3+}$ ion with $h_{x}=0.85 \mathrm{~K}$ and $A_{\mathrm{hf}}=0.319 \mathrm{~K}$. Green solid lines show a mean-field Monte Carlo calculation including spin-spin interactions $\left(J_{\mathrm{NN}}=-0.64 \mathrm{~K}\right.$, $D=1.29 \mathrm{~K}$ ). (b) Magnetic entropy change for $C_{m}$ from $20 \mathrm{~K}$ to the lowest measurement temperature of $76 \mathrm{mK}$ (colors and symbols as above). Our measurements on the SS sample render a residual entropy of $1.5(5) \mathrm{J} \mathrm{K}^{-1} \mathrm{~mol}_{\mathrm{Ho}}^{-1}$ at $87 \mathrm{mK}$, and our meanfield Monte Carlo simulations predict a zero-point entropy of $0.910 \mathrm{~J} \mathrm{~K}^{-1} \mathrm{~mol}_{\mathrm{Ho}}^{-1}$.

magnetic entropy reaches $21.5(5) \mathrm{J} \mathrm{K}^{-1} \mathrm{~mol}_{\mathrm{Ho}}^{-1}$, which is $6.5 \%$ smaller than the expectation of $R(\ln 2+\ln 8)=$ $23.05 \mathrm{~J} \mathrm{~K}^{-1} \mathrm{~mol}_{\mathrm{Ho}}^{-1}$ considering both the electronic and nuclear spin degrees of freedom [Fig. 3(b)] and assuming the absence of low-lying excited crystal-field states. Notwithstanding the systematic uncertainty arising from the large nuclear specific heat, the experimental residual entropy of $1.5(5) \mathrm{J} \mathrm{K}^{-1} \mathrm{~mol}_{\mathrm{Ho}}^{-1}$ at $87 \mathrm{mK}$ is comparable to the expected residual entropy of $0.92 \mathrm{~J} \mathrm{~K}^{-1} \mathrm{~mol}_{\mathrm{Ho}}^{-1}$ associated with the CSF degeneracy of the electronic spins $[38,41]$, consistent with a spin fragmentation picture in $\mathrm{Ho}_{3} \mathrm{Mg}_{2} \mathrm{Sb}_{3} \mathrm{O}_{14}$.

In Ho-based systems with a doublet single-ion ground state, such as $\mathrm{Ho}$ metal [65], $\mathrm{Ho}_{2} \mathrm{Ti}_{2} \mathrm{O}_{7}$ [78], and $\mathrm{LiHoF}_{4}$ [79], the nuclear specific heat consists of a broad peak (Schottky anomaly) below the ordering temperature of the electronic spins. This implies that the dynamics of the electronic and nuclear subsystems separate in such systems, with electronic spins already in their $\sigma^{z}$ eigenstate when nuclear spins start to follow them at low temperature.
This paradigm is not applicable to $\mathrm{Ho}_{3} \mathrm{Mg}_{2} \mathrm{Sb}_{3} \mathrm{O}_{14}$ because $h^{x}$ mixes $\left|+, I^{z}\right\rangle$ and $\left|-, I^{z}\right\rangle$ at each site [Fig. 2(d)]. Accordingly, the single-ion Hamiltonian [Eq. (7)] predicts two peaks for the nuclear specific heat; however, this model strongly disagrees with our experimental data [Fig. 3(a)]. The observed $C_{m}$ is also very different from that of unfrustrated TIM magnets such as $\mathrm{HoF}_{3}$, in which hyperfine interactions act as an effective mean field (MF) that precipitates long-range ordering of electronic spins, and the nuclear Schottky anomaly is only manifest below $T^{*}$ [80]. By contrast, in $\mathrm{Ho}_{3} \mathrm{Mg}_{2} \mathrm{Sb}_{3} \mathrm{O}_{14}$, more than half of the total magnetic entropy has already been recovered above $T^{*}$, suggesting the development of short-range spin correlations with a coupled electronic-nuclear character. Our heatcapacity measurements thus provide the first experimental hint of many-body physics in $\mathrm{Ho}_{3} \mathrm{Mg}_{2} \mathrm{Sb}_{3} \mathrm{O}_{14}$, demonstrating that the hyperfine term, transverse field, and spin-spin interactions in Eq. (10) must be treated on an equal footing.

\section{INELASTIC NEUTRON-SCATTERING MEASUREMENTS}

We use inelastic neutron-scattering measurements to probe the spin correlations and low-energy spin dynamics of $\mathrm{Ho}_{3} \mathrm{Mg}_{2} \mathrm{Sb}_{3} \mathrm{O}_{14}$. Neutron-scattering data as a function of momentum $(Q)$ and energy transfer $(\omega)$ are shown in Fig. 4(a) over the temperature range from 4.2 to $0.12 \mathrm{~K}$. The $Q$ dependence of the magnetic scattering shown in Fig. 4(b) was obtained by integrating over $-20 \leq \omega \leq$ $20 \mathrm{~K}$ and subtracting the paramagnetic data at $T=30 \mathrm{~K}$. Such energy-integrated data measure the Fourier transform of the instantaneous spin-pair correlation function. The energy dependence shown in Fig. 4(c) was obtained by integrating the inelastic scattering over $0.4 \leq Q \leq 1.6 \AA^{-1}$ and correcting for background scattering using emptycontainer measurements.

We first discuss the paramagnetic regime above $T^{*}$. For sample temperatures between 4.2 and $0.4 \mathrm{~K}$, the energyintegrated magnetic diffuse scattering displays a clear $Q$ dependence, with a broad peak centered at approximately $0.65 \AA^{-1}$ that develops on cooling [Fig. 4(b)]. This feature closely resembles observations for $\mathrm{Dy}_{3} \mathrm{Mg}_{2} \mathrm{Sb}_{3} \mathrm{O}_{14}$, where it was interpreted in terms of the development of kagome-ice correlations with a one in, two out or two in, one out ice rule on each triangle [41]. The energy-resolved response shows two main magnetic features: an intense and resolution-limited quasielastic peak and a broad inelastic tail extending to $\omega \approx 15 \mathrm{~K}$ that decays slowly with increasing energy transfer [Fig. 4(c)]. This energyresolved response differs dramatically from the case of $\mathrm{Dy}_{3} \mathrm{Mg}_{2} \mathrm{Sb}_{3} \mathrm{O}_{14}$, in which only elastic $(\omega=0)$ neutron scattering was observed at comparable temperatures, indicating time-independent spin correlations [41]. Moreover, the energy-resolved response of $\mathrm{Ho}_{3} \mathrm{Mg}_{2} \mathrm{Sb}_{3} \mathrm{O}_{14}$ is very different from the single-ion model discussed in Sec. III, in 

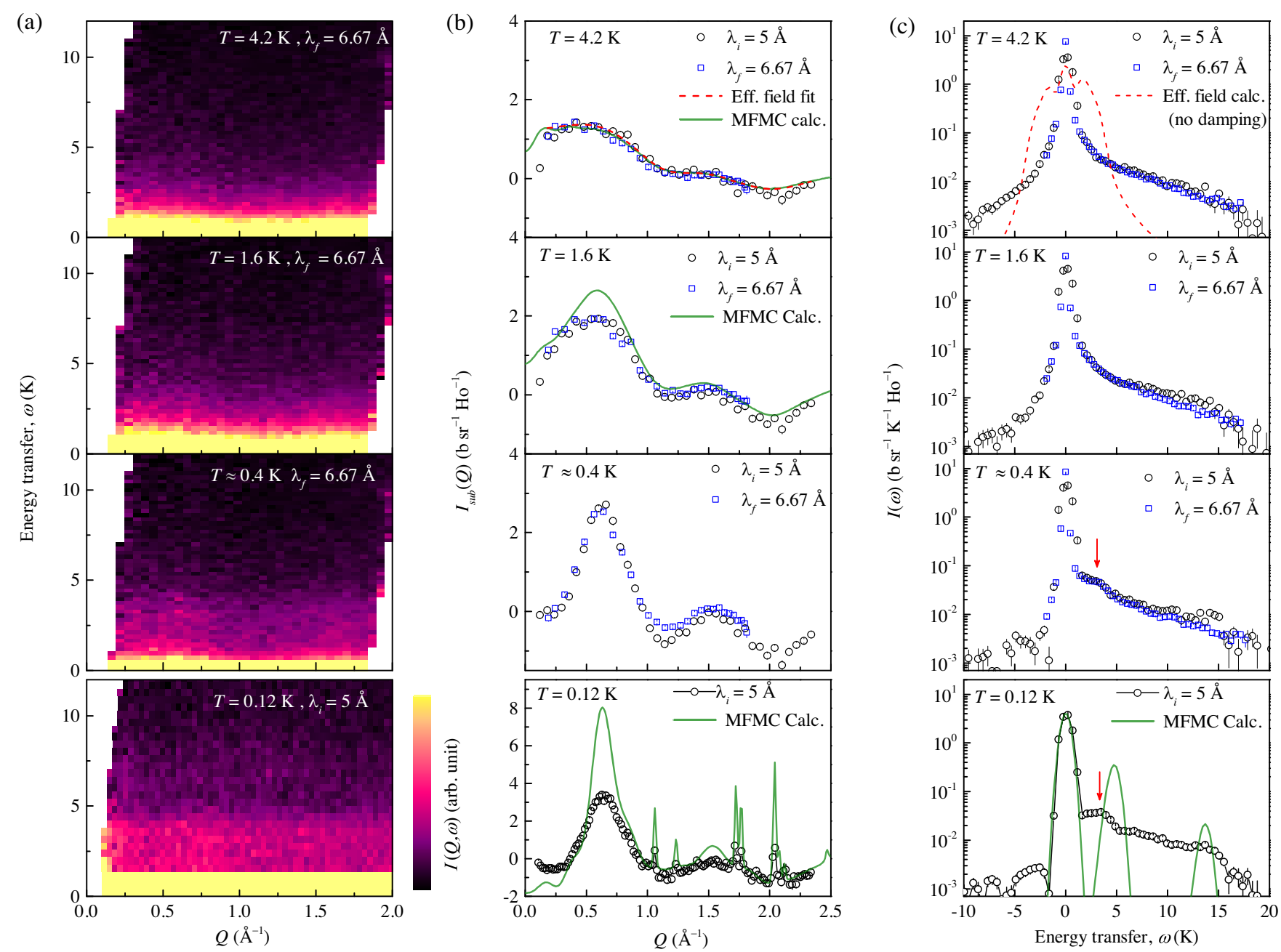

FIG. 4. Low-energy neutron-scattering response of $\mathrm{Ho}_{3} \mathrm{Mg}_{2} \mathrm{Sb}_{3} \mathrm{O}_{14}$ at $T=4.2,1.6,0.4$, and $0.12 \mathrm{~K}$ (top to bottom panels). Data are from two distinct measurements on different spectrometers and samples. Black circles correspond to measurements on the SS sample using the DCS spectrometer with an incident neutron wavelength of $5 \AA$; blue squares correspond to measurements on the SG sample using the OSIRIS spectrometer with a final neutron wavelength of $6.67 \AA$. (a) Dependence of the inelastic magnetic scattering intensity $I(Q, \omega)$ on wave-vector transfer $Q$ and energy transfer $\omega$. (b) Energy-integrated $(-20 \leq \omega \leq 20 \mathrm{~K})$ magnetic neutron-scattering intensity $I_{\text {sub }}(Q)$ at four temperatures, showing experimental data (black circles), fits using the paramagnetic effective field theory (dashed red lines), and calculations using the mean-field theory (solid olive lines). The correlated magnetic scattering has been isolated by subtracting high-temperature data or simulations taken at $T=30 \mathrm{~K}$, which leads to negative values for the subtracted intensity. (c) Energy dependence of magnetic neutron-scattering intensity $I(\omega)$ integrated over wave-vector transfers $0.4 \leq Q \leq 1.6 \AA^{-1}$ at four different temperatures. An empty container subtraction was used to remove background.

which the majority of scattering is expected to be inelastic and concentrated around energy transfer $\Delta=2 h^{x} \approx 1.7 \mathrm{~K}$ [Figs. 2(e) and 2(f)]. The most likely reason for this discrepancy is the presence of significant pairwise interactions in $\mathrm{Ho}_{3} \mathrm{Mg}_{2} \mathrm{Sb}_{3} \mathrm{O}_{14}$. Indeed, theoretical work has shown that a strong damping of inelastic excitations is intrinsic to the TIM, and is strongest for $J_{i j} \approx h^{x}[81-83]$. Experiments on model two-singlet systems such as $\mathrm{LiTbF}_{4}$ are qualitatively consistent with this picture [84-86]. Hence, our inelastic neutron-scattering results support a picture of $\mathrm{Ho}_{3} \mathrm{Mg}_{2} \mathrm{Sb}_{3} \mathrm{O}_{14}$ in which frustrated pairwise interactions compete with quantum fluctuations induced by $h^{x}$.
To obtain a better understanding of our paramagnetic neutron-scattering data, we employ a reciprocal-space mean-field approximation to model the wave-vector dependence of the magnetic diffuse scattering. This approach is exact in the high-temperature limit, and introduces the effect of local spin correlations via a reaction-field term that is determined self-consistently [87]. The neutron-scattering intensity is calculated via the dynamical susceptibility, which is approximated as

$$
\chi_{\mu}(\mathbf{Q}, \omega)=\frac{\chi_{0}(\omega)}{1-\chi_{0}(\omega)\left[\lambda_{\mu}(\mathbf{Q})-\lambda\right]}
$$


where $\chi_{0}(\omega)$ is the single-ion susceptibility obtained by exact diagonalization of $H_{\mathrm{SI}}, \lambda$ is the reaction field $[87,88]$, and $\mu \in\{1,3\}$ labels the normal modes of the Ising system in the same way as for classical mean-field theories [89]. Full details of this method are given in Ref. [87], and references therein. We employ this approach to fit the value of $J_{\mathrm{NN}}$ to the energy-integrated magnetic diffuse scattering. The value of $J_{\mathrm{NN}}$ is the only free parameter because $h^{x}=0.85 \mathrm{~K}$ is fixed (see Sec. III B). Our fits yield good agreement with the energy-integrated experimental data at 4.2 K [Fig. 4(b)] and at $10 \mathrm{~K}$ (not shown). However, because damping effects are not included in the mean-field calculation, the energy-resolved response deviates from the experimental results [Fig. 4(c)]. The qualitative features of our calculations are not strongly sensitive to the value of $J_{\mathrm{NN}}$, provided that the interactions between pseudospins remain frustrated. Nevertheless, $J_{\mathrm{NN}}=-0.64(4) \mathrm{K}$ yields optimal fits, and we therefore use this value for calculations in the rest of this paper.

We now consider the low-temperature state below $T^{*}$. In this regime, most of the magnetic neutron-scattering intensity remains diffuse; however, weak magnetic Bragg peaks also appear on top of the magnetic diffuse scattering [Fig. 4(b)]. The wave-vector dependence of the scattering closely resembles observations for the $\mathrm{Dy}_{3} \mathrm{Mg}_{2} \mathrm{Sb}_{3} \mathrm{O}_{14}$, suggesting that a similar spin fragmentation process occurs in $\mathrm{Ho}_{3} \mathrm{Mg}_{2} \mathrm{Sb}_{3} \mathrm{O}_{14}$. In particular, the divergence-full part of a spin-fragmented state describes an all in, all out long-range magnetic ordering involving only a small fraction $(\Sigma \mu / 3)$ of the total magnetic moment [Fig. 1(a)]. Rietveld refinements to the weak magnetic Bragg component of our $T=0.12 \mathrm{~K}$ data are in good agreement with the AIAO average magnetic structure in $\mathrm{Ho}_{3} \mathrm{Mg}_{2} \mathrm{Sb}_{3} \mathrm{O}_{14}$, consistent with a spin-fragmented state (see Appendix A). Our model calculations, discussed in Sec. VI, provide further evidence that a spin-fragmented state is consistent with the spin Hamiltonian, Eq. (10).

However, our measurements also reveal fundamental differences with the CSF state observed in $\mathrm{Dy}_{3} \mathrm{Mg}_{2} \mathrm{Sb}_{3} \mathrm{O}_{14}$. First, the observed magnetic Bragg intensities in $\mathrm{Ho}_{3} \mathrm{Mg}_{2} \mathrm{Sb}_{3} \mathrm{O}_{14}$ are strongly reduced compared to the expected classical value. The magnetic Bragg intensity is proportional to the square of the ordered magnetic moment, which is $\mu / 3=3.3 \mu_{B}$ per site for a CSF state in the absence of chemical disorder (Fig. 1) $[40,41]$. In contrast, Rietveld refinements to our $T=0.12 \mathrm{~K}$ data indicate an ordered magnetic moment of only $1.70(3) \mu_{B}$ per $\mathrm{Ho}^{3+}$ (see Appendix A). Importantly, $\mathrm{Dy}_{3} \mathrm{Mg}_{2} \mathrm{Sb}_{3} \mathrm{O}_{14}$ has both a larger ordered moment [2.66(6) $\mu_{B}$ per Dy $^{3+}$ at $0.20 \mathrm{~K}$ [41]] and a greater degree of site mixing than $\mathrm{Ho}_{3} \mathrm{Mg}_{2} \mathrm{Sb}_{3} \mathrm{O}_{14}$ (see Sec. III). This suggests that chemical disorder cannot fully explain the observed reduction in ordered moment in $\mathrm{Ho}_{3} \mathrm{Mg}_{2} \mathrm{Sb}_{3} \mathrm{O}_{14}$, although it may be a contributing factor. Given the low temperature of our measurement $(\approx 0.12 \mathrm{~K})$, quantum fluctuations are the most likely alternative explanation.
The second key difference with $\mathrm{Dy}_{3} \mathrm{Mg}_{2} \mathrm{Sb}_{3} \mathrm{O}_{14}$ is that the spin-fragmented state in $\mathrm{Ho}_{3} \mathrm{Mg}_{2} \mathrm{Sb}_{3} \mathrm{O}_{14}$ is accompanied by the persistence of continuous magnetic excitations down to at least $0.12 \mathrm{~K}$. Furthermore, a distinct mode develops at $\omega \approx 3 \mathrm{~K}$ when approaching $T^{*}$ and appears clearly separated from the elastic line in $I(Q, \omega)$ below $T^{*}$ [red arrows in Fig. 4(c)]. Above this mode, we observe a high-energy tail extending to $\omega \approx 15 \mathrm{~K}$ that resembles the slow decay from the central peak in the paramagnetic phase. The presence of clear low-temperature spin dynamics over a wide energy range strongly contrasts with canonical classical Ising magnets such as $\mathrm{Ho}_{2} \mathrm{Ti}_{2} \mathrm{O}_{7}$ [90] and $\mathrm{Dy}_{3} \mathrm{Mg}_{2} \mathrm{Sb}_{3} \mathrm{O}_{14}$ [41], in which the spin dynamics are too slow to observe in neutron-scattering measurements at comparable temperatures [43]. Meanwhile, spin fragmentation accompanied by inelastic excitations has been observed in $\mathrm{Nd}_{2} \mathrm{Zr}_{2} \mathrm{O}_{7}$ [21,91], yet with a key difference that reflects their different physical origins: unlike $\mathrm{Nd}_{2} \mathrm{Zr}_{2} \mathrm{O}_{7}$ where pinch points appear only in the inelastic channel, the diffuse scattering observed in $\mathrm{Ho}_{3} \mathrm{Mg}_{2} \mathrm{Sb}_{3} \mathrm{O}_{14}$ contains both elastic and inelastic contributions, most of which is elastic within our highest energy resolution of approximately $0.29 \mathrm{~K}(25 \mu \mathrm{eV})$ FWHM on OSIRIS [Fig. 4(c)]. Overall, the enhancement of inelastic scattering and reduction of the magnetic Bragg intensity provides experimental evidence for quantum excitations above a spin-fragmented ground state in $\mathrm{Ho}_{3} \mathrm{Mg}_{2} \mathrm{Sb}_{3} \mathrm{O}_{14}$.

\section{THEORETICAL MODELING}

Our experimental results have revealed two key insights: that the spin Hamiltonian of $\mathrm{Ho}_{3} \mathrm{Mg}_{2} \mathrm{Sb}_{3} \mathrm{O}_{14}$ realizes a frustrated transverse Ising model, and that a spinfragmented state with quantum spin dynamics exists in $\mathrm{Ho}_{3} \mathrm{Mg}_{2} \mathrm{Sb}_{3} \mathrm{O}_{14}$ at the lowest measurable temperatures. These results identify $\mathrm{Ho}_{3} \mathrm{Mg}_{2} \mathrm{Sb}_{3} \mathrm{O}_{14}$ as an exotic frustrated magnet with unconventional properties. However, two important questions remain. First, how do frustrated interactions, transverse field, and hyperfine coupling conspire to generate the observed quantum spin dynamics? And, second, are these quantum dynamics primarily singlesite fluctuations, or do they involve many-body quantum correlations as hypothesized for the QSF state in the Introduction?

As a first step toward answering these questions, we perform a theoretical analysis of the spin Hamiltonian, Eq. (10). Because this Hamiltonian describes a long-range interacting quantum system with several interactions on the same energy scale, no single theoretical approach can provide a complete description of its properties. We therefore employ two complementary approaches that ultimately reveal how the hyperfine coupling interplays with the transverse field. First, in Sec. VI A, we use exact diagonalization of small clusters to investigate possible quantum correlations between sites. Second, in Sec. VIB, we develop a modification of Monte Carlo simulation in 
conjunction with real-space mean-field theory, which enables us to investigate finite-temperature properties on large clusters, for which quantum effects are considered only at the single-site level.

\section{A. Exact diagonalization}

We use exact diagonalization of small clusters to explore the ground state and low-energy spectrum of the model Hamiltonian, Eq. (10). The main results presented here are obtained from diagonalizing a $2 \times 2$ rhombus-shaped supercell with $N=12$ sites; however, our conclusions are robust to the choice of different supercell geometries and the use of 18-site clusters (see Appendix E).

We start by considering a simple quantum model that contains the essence of $\mathrm{Ho}_{3} \mathrm{Mg}_{2} \mathrm{Sb}_{3} \mathrm{O}_{14}$ : a dipolar kagome ice under a transverse field. In the absence of the hyperfine and exchange couplings, the system is controlled by a single tuning parameter $h^{x} / D$. At the classical point $\left(h^{x} / D=0\right), 12$ CSF configurations appear to be exactly degenerate for the $2 \times 2$ cluster. Given that this model is known to order completely in the thermodynamic limit, with a $\sqrt{3} \times \sqrt{3}$ enlargement of the unit cell [38] that matches our rhombus-shaped finite system, these degenerate states are the six possible domains of the fully ordered phase plus their time-reversal symmetric counterparts. Distinguishing a CSF phase from this fully ordered ground state requires larger size clusters, because the degeneracy of the former grows exponentially with system size while the degeneracy of the latter is fixed.

For the same cluster, an infinitesimal transverse field yields a unique ground state, corresponding to a quantum superposition of the above $12 \mathrm{CSF}$ spin configurations with equal weights, and opens a gap [Fig. 5(a)]. The energy gap to the first excited state scales as $\left(h^{x} / D\right)^{6}$ until $h^{x} / D \lesssim$ 0.2 [Fig. 5(a)], reflecting that the leading-order quantum tunneling process between degenerate CSF states corresponds to flipping six spins around a hexagon (Fig. 1). Our calculations show that the mixing of states out of the CSF manifold is negligible when $h^{x} / D$ is small, which can be seen by projecting the ground-state wave function into the CSF subspace [Fig. 5(b)]. As the transverse field is increased, we see a dramatic decrease of the overlap between the ground state and the CSF manifold at $h^{x} / D \approx 0.2$, accompanied by a change in the scaling behavior of the energy gap and a peak in the second derivative of the ground-state energy [Fig. 5(a)]. Notably, these features are absent in a transverse Ising model with only nearest-neighbor exchange interactions (see Fig. 9), implying possible existence of a quantum phase transition when the long-range dipolar interaction is included in the model. The system eventually crosses over into a paramagnetic phase in the limit $h^{x} \gg D$, corresponding to the limit of isolated single-ion physics.

The above results seem to point to a possible existence of a QSF phase when the transverse field remains a
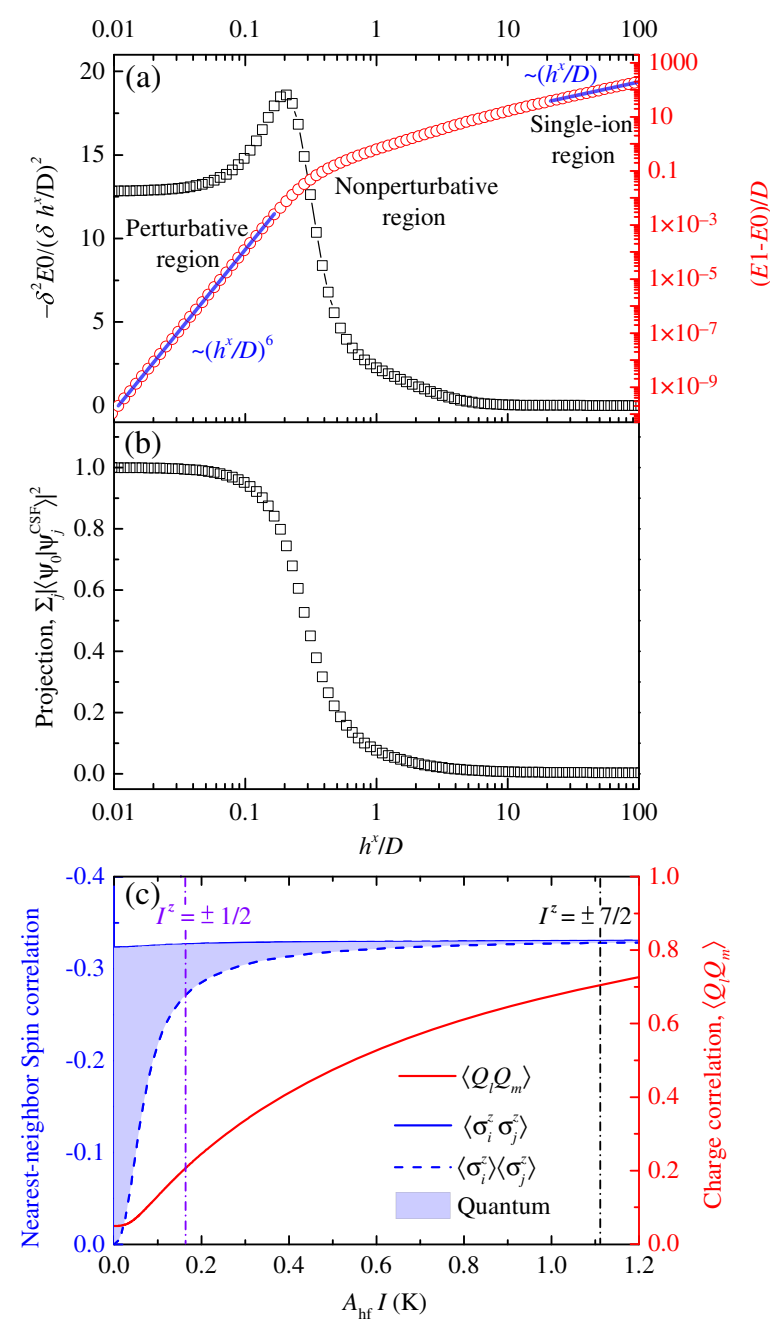

FIG. 5. Results of exact diagonalization of the effective Hamiltonian [Eq. (10)] on an $N=12$ cluster. (a) and (b) are obtained with $J_{\mathrm{NN}}=0$ and $A_{\mathrm{hf}}=0$. (a) Second derivative of the ground-state energy as a function of $h^{x} / D$ (black squares) and energy gap between the ground state and first excited state (red circles), indicating possible quantum phase transitions. Blue lines represent linear fits to the data. (b) Projection of the ground-state wave function into the CSF basis (black squares). (c) Nearestneighbor spin correlation (blue) and emergent charge correlation (red) of the ground states as a function of hyperfine coupling strength where $Q_{l}$ and $Q_{m}$ are the emergent charges defined as in Fig. 1. Parameters appropriate for $\mathrm{Ho}_{3} \mathrm{Mg}_{2} \mathrm{Sb}_{3} \mathrm{O}_{14}(D=1.29 \mathrm{~K}$, $J_{\mathrm{NN}}=-0.64 \mathrm{~K}, h^{x}=0.85 \mathrm{~K}$ ) are used in (c). The two dashed lines indicate nuclear spin sectors with $I_{i}^{z}= \pm 1 / 2$ and $\pm 7 / 2$ on each site, respectively.

perturbation to the dipolar interaction. However, a recent quantum Monte Carlo (QMC) work has uncovered that the ground state of a very related system (with spins in plane rather than tilted by $22^{\circ}$ ) displays a $\sqrt{3} \times \sqrt{3}$ magnetic order up to at least $h^{x} / D \lesssim 0.65$ [49]. For such an ordered state, ED will yield a quantum superposition of magnetic domains, so that we cannot distinguish between long-range magnetic order and genuine quantum superposition state 
without size-scaling studies and consideration of larger size clusters. We also note that even if a quantum superposition state was observed for larger clusters, the CSF subspace should be divided into disconnected topological sectors [92] where the composition of the wave functions depends on the details of the topology. Whether the aforementioned quantum phase transition at finite $h^{x}$ exists in the thermodynamic limit requires further study.

Notwithstanding these limitations, we can use ED on small clusters to identify the effect of hyperfine coupling as relevant for $\mathrm{Ho}_{3} \mathrm{Mg}_{2} \mathrm{Sb}_{3} \mathrm{O}_{14}$. We proceed as follows. First, we put Eq. (10) into a block-diagonal form where each block has a set of fixed nuclear spin numbers $\left\{I_{1}^{z}, I_{2}^{z}, \ldots, I_{N}^{z}\right\}$ and the hyperfine term is treated as a site-dependent local longitudinal field; this is possible because $I^{z}$ remains a good quantum number. Then, we find the global ground state of the system by diagonalizing the Hamiltonian in each of the $8^{N}$ nuclear spin blocks independently. The interplay between hyperfine coupling and quantum tunneling is shown in Fig. 5(c). Here, we use the parameters $J_{\mathrm{NN}}=-0.64 \mathrm{~K}, h^{x}=0.85 \mathrm{~K}$, and $D=1.29 \mathrm{~K}$ relevant for $\mathrm{Ho}_{3} \mathrm{Mg}_{2} \mathrm{Sb}_{3} \mathrm{O}_{14}$, and plot the nearest-neighbor spin and charge correlation functions as a function of the hyperfine coupling energy scale $A_{\mathrm{hf}} I$. In the absence of hyperfine coupling, the ground state is in the nonperturbative regime of our ED calculations with strong correlations between spins. Although the exact nature of the ground state in this regime requires further investigation, ED remains informative to track the effect of hyperfine interactions. For $A_{\mathrm{hf}} I=1.11 \mathrm{~K}$, as appropriate for $\mathrm{Ho}_{3} \mathrm{Mg}_{2} \mathrm{Sb}_{3} \mathrm{O}_{14}$, the ground state has $I_{i}^{z}= \pm 7 / 2$ for every site. Upon increasing $A_{\mathrm{hf}}$, the nearest-neighbor quantum correlation-defined as the total correlation minus the classical correlation, $\left\langle\sigma_{i}^{z} \sigma_{j}^{z}\right\rangle-\left\langle\sigma_{i}^{z}\right\rangle\left\langle\sigma_{j}^{z}\right\rangle$-diminishes quickly from $100 \%$ at $A_{\mathrm{hf}} I=0$ to only $1 \%$ of the total correlation for $A_{\mathrm{hf}} I=1.11 \mathrm{~K}$ [Fig. 5(c)]. This result implies that if quantum correlations are present in the ground state of $\mathrm{Ho}_{3} \mathrm{Mg}_{2} \mathrm{Sb}_{3} \mathrm{O}_{14}$, they are dramatically suppressed by hyperfine interactions. As the system is thermally excited to hyperfine levels with smaller $\left|I^{z}\right|$, quantum correlations may reappear [Fig. 5(c)].

Overall, our ED results suggest that the low-temperature physics of $\mathrm{Ho}_{3} \mathrm{Mg}_{2} \mathrm{Sb}_{3} \mathrm{O}_{14}$ can be understood in terms of a semiclassical ground state without quantum correlations between sites and predominantly single-ion quantum fluctuations. However, we speculate that enhanced quantum correlations may appear in the system when other hyperfine states are populated at elevated temperatures.

\section{B. Mean-field Monte Carlo simulations}

Having established exact results for small spin clusters, we now develop a numerical method capable of approximating local quantum fluctuations in large spin configurations. The essence of our approach is to combine Monte Carlo simulations and exact diagonalization of the mean-field Hamiltonian at each site to obtain equilibrium configurations of static spins.

We begin by summarizing our approach, which we term "mean-field Monte Carlo" (MFMC). The mean-field Hamiltonian of a given site $i$ in a spin configuration is given by

$$
\mathcal{H}_{i}=h_{i}^{z} \sigma_{i}^{z}+h^{x} \sigma_{i}^{x}
$$

where

$$
h_{i}^{z}=A_{\mathrm{hf}} I_{i}^{z}+\sum_{j} J_{i j}\left\langle\sigma_{j}^{z}\right\rangle
$$

is the longitudinal field that includes contributions from exchange, dipolar, and hyperfine interactions. Equation (12) describes a $2 \times 2$ matrix in each sector $n \in\{1, \ldots, 8\}$ of the nuclear spin $I_{i}^{z}$. Diagonalizing these matrices yields the single-ion eigenstates in the same form as those shown in Fig. 2(d), i.e., $\left|\Psi_{i}\right\rangle_{n}=\left|\psi_{i}^{ \pm}, I_{i}^{z}\right\rangle$. We obtain the static spin on site $i$ as the diagonal matrix element of $\sigma_{i}^{z}$,

$$
\left\langle\sigma_{i}^{z}\right\rangle=\left\langle\psi_{i}^{ \pm}, I_{i}^{z}\left|\sigma_{i}^{z}\right| \psi_{i}^{ \pm}, I_{i}^{z}\right\rangle=\frac{ \pm h_{i}^{z}}{\sqrt{\left(h^{x}\right)^{2}+\left(h_{i}^{z}\right)^{2}}} .
$$

For zero transverse field, the static spin $\left\langle\sigma_{i}^{z}\right\rangle$ is a classical Ising variable with unit magnitude on all sites. For nonzero transverse field, $\left\langle\sigma_{i}^{z}\right\rangle$ has a magnitude of less than unity, reflecting the nonzero probability of transverse-field-induced spin flips. The probability amplitudes of such spin flips are given by the off-diagonal matrix elements,

$$
\left\langle\psi_{i}^{ \pm}, I_{i}^{z}\left|\sigma_{i}^{z}\right| \psi_{i}^{\mp}, I_{i}^{z}\right\rangle=\frac{-h^{x}}{\sqrt{\left(h^{x}\right)^{2}+\left(h_{i}^{z}\right)^{2}}} .
$$

To take account of this effect, in our Monte Carlo simulation we do not enforce $\left\langle\sigma_{i}^{z}\right\rangle= \pm 1$; instead, we compute $\left\langle\sigma_{i}^{z}\right\rangle$ on the fly from mean-field states $\left|\psi_{i}^{ \pm}, I_{i}^{z}\right\rangle$. Our protocol is as follows. We initialize $\left\langle\sigma_{i}^{z}\right\rangle$ with random uniformly distributed values between -1 and 1. One MC step consists of selecting a random site $i$ and proposing an update to the nuclear spin quantum number $\left(I_{i}^{z} \rightarrow \tilde{I}_{i}^{z}\right)$ and electronic static spin, with the latter selected at random from three possibilities: (i) maintaining the static spin $\left(\left\langle\sigma_{i}^{z}\right\rangle \rightarrow\left\langle\sigma_{i}^{z}\right\rangle\right)$, (ii) flipping the static spin $\left(\left\langle\sigma_{i}^{z}\right\rangle \rightarrow-\left\langle\sigma_{i}^{z}\right\rangle\right)$, and (iii) going to one of the two new mean-field states obtained by diagonalizing the mean-field Hamiltonian, Eq. (12), for which both length and direction of the static spin may be changed $\left(\left\langle\sigma_{i}^{z}\right\rangle \rightarrow\left\langle\tilde{\sigma}_{i}^{z}\right\rangle\right)$. The move is accepted or rejected according to the Metropolis protocol (see Appendix F). Our method is identical to a classical MC simulation with single spin-flip moves in the limit of vanishing transverse field.

As in Sec. VI A, we begin by considering the simple case with $A_{\mathrm{hf}}=0$. Figure 6(a) shows a MFMC phase diagram as a function of $h^{x} / D$ and $J_{\mathrm{NN}} / D$ at low temperature 


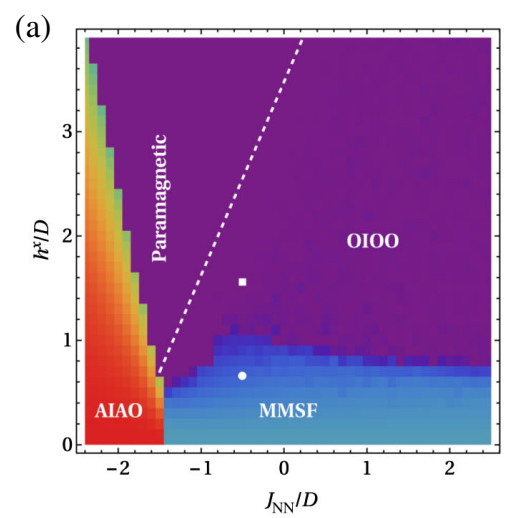

(d)

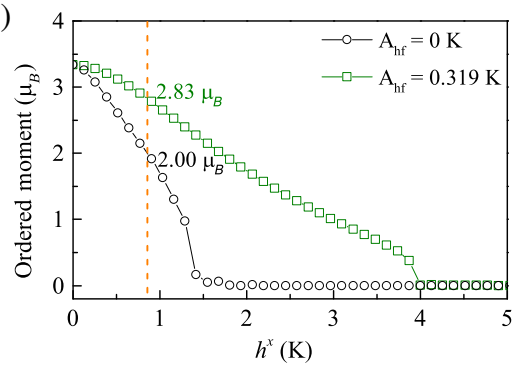

(b)
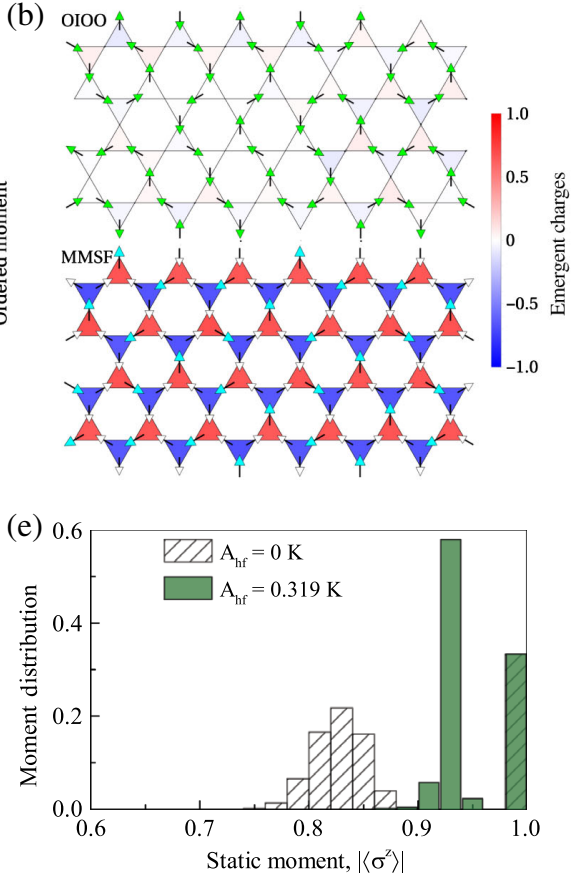
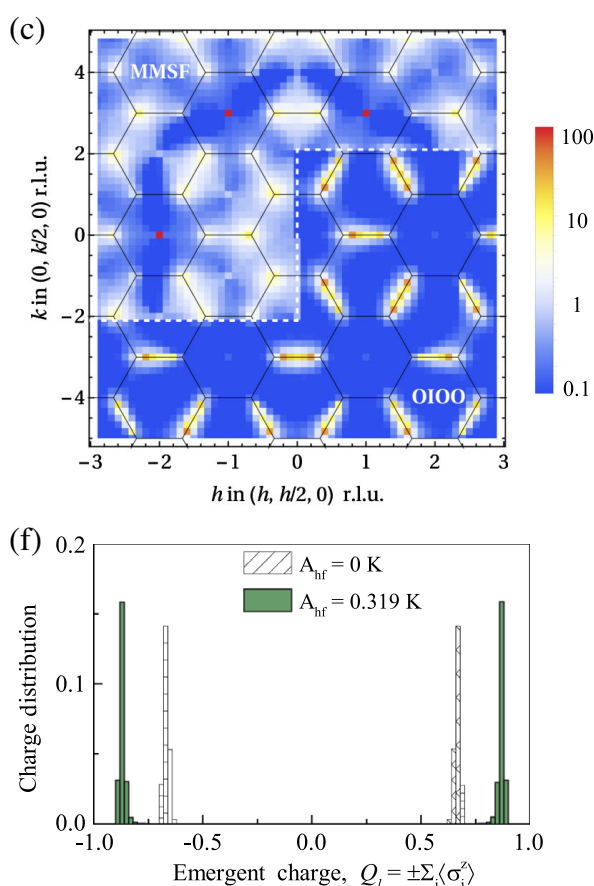

FIG. 6. Mean-field Monte Carlo (MFMC) simulation results of the mean-field Hamiltonian, Eq. (12). (a) Phase diagram as a function of $J_{\mathrm{NN}} / D$ and $h^{x} / D$ with $A_{\mathrm{hf}}=0$. The color represents the ordered moment of the all in, all out (AIAO) structure at $0.12 \mathrm{~K}$. Four distinct phases are observed: AIAO, paramagnetic, one in, one out (OIOO), and moment-modulated spin fragmented (MMSF). The boundary (white dashed line) between the paramagnetic phase and the OIOO phase is obtained from the phase diagram of the static moment (see Fig. 11). (b) Representative spin configurations of the MMSF phase and the OIOO phase, with $h^{x}$ chosen to be $0.85 \mathrm{~K}$ [white dot in (a)] and $2 \mathrm{~K}$ [white square in (a)], respectively. The exchange interaction $J_{\mathrm{NN}}=-0.64 \mathrm{~K}$ and the dipolar interaction $D=1.29 \mathrm{~K}$ are the same for all subsequent panels in this figure. The sizes of arrows are scaled with static spins $\left\langle\sigma_{i}^{z}\right\rangle$ on each site. Cyan and white arrows denote long $\left(\left|\left\langle\sigma_{i}^{z}\right\rangle\right|>0.9\right)$ and short static spins $\left(\left|\left\langle\sigma_{i}^{z}\right\rangle\right|<0.9\right.$ ), respectively. (c) Fourier transform of static spin correlations $\left\langle\left\langle\sigma_{i}^{z}\right\rangle\left\langle\sigma_{j}^{z}\right\rangle\right\rangle$ in the MMSF phase (top) and the OIOO phase (bottom). (d) Ordered moment as a function of $h^{x}$ with and without hyperfine coupling (green squares and black circles, respectively). The orange line corresponds to $h^{x}=0.85 \mathrm{~K}$ for $\mathrm{Ho}_{3} \mathrm{Mg}_{2} \mathrm{Sb}_{3} \mathrm{O}_{14}$. (e) Normalized distribution of static spin lengths and (f) normalized distribution of magnetic charges for the MMSF phase with and without hyperfine couplings (green and black bars, respectively).

$(T=0.12 \mathrm{~K})$. We observe two trivial phases: first, for dominating $h^{x}$, a paramagnetic ground state with $\left\langle\sigma_{i}^{z}\right\rangle=0$ is obtained as anticipated, and, second, for small $h^{x}$ and nonfrustrated interactions $\left(J_{\mathrm{NN}} / D \ll 0\right)$, a conventional AIAO order is obtained, in which the mean field has the same magnitude on all sites. In contrast, frustrated interactions $\left(J_{\mathrm{NN}} / D \gtrsim-1.5\right)$ favor nontrivial states in which the mean field-and hence the static spin-is spatially modulated [93]. Depending on the relative strength of $h^{x}$ compared to the pairwise interactions, this stabilizes two distinct phases. For $h^{x} \gtrsim D$, we find a spin-liquid-like "one in, one out" (OIOO) phase characterized by a local constraint on every triangle: two out of the three spins are static with roughly equal moments and trace out closed loops in the kagome planes, while the third spin remains entirely dynamic due to the local cancellation of mean fields; hence, emergent magnetic charges are absent [Fig. 6(b)]. Spin correlations of the OIOO phase give rise to distinct starlike signature on Brillouin zone edges in momentum space, while magnetic Bragg peaks and pinchpoint features associated with a spin-fragmented state are absent [Fig. 6(c)]. For $h^{x} \lesssim D$, we find a phase resembling a CSF state but dressed with a static moment modulation from site to site; we call this phase moment-modulated spin fragmented (MMSF). In this state, quantum fluctuations are manifest for each triangle in the form of one "long" static spin with a large magnitude, and two "short" static spins with smaller magnitudes [Fig. 6(b)]. Remarkably, despite a broad distribution of the static spin length [Fig. 6(e)], sharp magnetic Bragg peaks and diffuse scattering with pinchpoint-like features coexist in momentum space [Fig. 6(c)]. This observation provides a "smoking gun" for spin fragmentation, which can be understood in the following way. Because each triangle has a one in, two out or two in, one out arrangement of static spins, it supports well-defined emergent magnetic charges, $\left\langle Q_{k}\right\rangle= \pm \sum_{i \in k}\left\langle\sigma_{i}^{z}\right\rangle$, where $i$ runs over three spins in a triangle $k$. Importantly, $\left\langle Q_{k}\right\rangle$ form a staggered order with a small variation in magnitude [Figs. 6(b) and 6(f)]. In the language of spin fragmentation, once we separate the divergence-full part of the MMSF phase (equivalent to the average value of $\left\langle Q_{k}\right\rangle$ or the ordered moment of the AIAO structure), the remaining part 
is approximately divergence free and hence gives rise to diffuse scattering with pinch-point singularities. In some sense, the MMSF state is a mean-field mimic of the QSF state where all the quantum many-body correlations are approximated by classical ones.

We now compare our experimental data with MFMC calculations using the parameters $D=1.29 \mathrm{~K}, h^{x}=0.85 \mathrm{~K}$, and $J_{\mathrm{NN}}=-0.64 \mathrm{~K}$ appropriate for $\mathrm{Ho}_{3} \mathrm{Mg}_{2} \mathrm{Sb}_{3} \mathrm{O}_{14}$. For $A_{\mathrm{hf}}=0$, our MFMC simulations predict a MMSF phase at low temperature [Fig. 6(a)]. Setting $A_{\mathrm{hf}}$ to the experimental value of $0.319 \mathrm{~K}$ enhances the classical spin correlations of the MMSF state and increases the magnitudes of the static moments, ordered moments, and emergent magnetic charges [Figs. 6(d)-6(f)]. Our calculated heat capacity shows a significant improvement over the singleion calculation and agrees qualitatively with the experimental data (Fig. 3). The calculation captures three major features: a broad shoulder between 1 and $10 \mathrm{~K}$, a sharp phase transition into the spin-fragmented phase below $1 \mathrm{~K}$, and a large nuclear heat-capacity anomaly centered around $0.3 \mathrm{~K}$. Our calculated diffuse-scattering patterns agree quantitatively with our energy-integrated neutron-scattering data at $4.2 \mathrm{~K}$, and agree qualitatively with the measured momentum dependence of Bragg and diffuse scattering at $0.12 \mathrm{~K}$ [Fig. 4(b)]. However, quantitative discrepancies with experiment become increasingly significant as the simulation temperature is lowered: most notably, at $0.12 \mathrm{~K}$, the calculation is much sharper than the experimental data and strongly overestimates the intensities of the magnetic Bragg peaks. While the MFMC calculated ordered moment of $2.85 \mu_{B}$ at $0.12 \mathrm{~K}$ is in good agreement with the ED result of $2.80 \mu_{B}$, these values are considerably larger than the experimental result of $1.70(3) \mu_{B}$ [Fig. 6(d)]. There are two likely explanations for this discrepancy. First, our models neglect the effect of $\mathrm{Mg} / \mathrm{Ho}$ site disorder, but in fact approximately $3 \%$ of $\mathrm{Ho}$ sites are occupied by $\mathrm{Mg}$ in $\mathrm{Ho}_{3} \mathrm{Mg}_{2} \mathrm{Sb}_{3} \mathrm{O}_{14}$. Classical calculations suggest that this degree of site mixing can suppress the ordered moment by $\sim 0.5 \mu_{B}$ [41], i.e., by a similar amount to quantum fluctuations. Second, the large specific-heat anomaly at low temperature makes thermalization of the sample challenging, despite following experimental best practices (Sec. II). According to our MFMC calculations, if the true sample temperature is about $85 \%$ of $T^{*}$, the ordered moment will be suppressed to the experimental value in the absence of site disorder.

Although our MFMC calculations cannot describe collective excitations, the density of states of single-ion excitations shown in Fig. 4(c) provides insight into the dynamics of the MMSF state (see Appendix F). While such excitations are prohibited at low temperature in $\mathrm{Dy}_{3} \mathrm{Mg}_{2} \mathrm{Sb}_{3} \mathrm{O}_{14}$ due to the vanishing off-diagonal neutron dipolar matrix elements, this is no longer the case in $\mathrm{Ho}_{3} \mathrm{Mg}_{2} \mathrm{Sb}_{3} \mathrm{O}_{14}$ due to the presence of the transverse field [Eq. (15)]. In addition to a large central peak, our calculation shows two small inelastic peaks centered around $\omega=5$ and $14 \mathrm{~K}$ that correspond to flipping short and long static spins, respectively. The relative amount of spectral weight in the elastic versus inelastic channels is qualitatively consistent with the experimental data, but the distinct shoulder in the data appears roughly $3 \mathrm{~K}$ lower in energy transfer than in the calculation, and the calculation does not reproduce the continuous nature of the excitations. These observations may be a consequence of quantum correlations for the excited states, which are suggested by the ED results (Sec. VI A) but not included in the MFMC model; hence, quantum calculations of the excitation spectrum would be interesting to explore in future studies.

\section{DISCUSSION AND CONCLUSIONS}

Our study demonstrates that $\mathrm{Ho}_{3} \mathrm{Mg}_{2} \mathrm{Sb}_{3} \mathrm{O}_{14}$ realizes a frustrated quantum Ising magnet on kagome lattice with three competing energy scales: pairwise interactions between electronic spins, quantum tunneling via an intrinsic transverse field, and hyperfine coupling between electronic and nuclear spins. Our experiments uncover overdamped paramagnetic spin dynamics at high temperature and a spin-fragmented state with reduced ordered moment and enhanced single-ion quantum fluctuations at low temperature. The key difference with the CSF phase reported in isostructural $\mathrm{Dy}_{3} \mathrm{Mg}_{2} \mathrm{Sb}_{3} \mathrm{O}_{14}$ is the observable low-energy spin dynamics in $\mathrm{Ho}_{3} \mathrm{Mg}_{2} \mathrm{Sb}_{3} \mathrm{O}_{14}$. These dynamics are a consequence of the intrinsic transverse field emerging from the lower crystallographic symmetry of the $\mathrm{Ho}^{3+}$ site in $\mathrm{Ho}_{3} \mathrm{Mg}_{2} \mathrm{Sb}_{3} \mathrm{O}_{14}$ compared to pyrochlore spin ices. Crucially, the transverse field is homogeneous, in contrast to random fields induced by chemical disorder. Therefore, a wide-ranging implication of our study is that symmetry lowering need not be a complicating factor in condensed-matter systems, but can instead enable the observation of simple models of quantum frustration.

Our results motivate further theoretical investigations of the effect of quantum fluctuations on partially ordered states, such as the spin fragmentation observed here for $\mathrm{Ho}_{3} \mathrm{Mg}_{2} \mathrm{Sb}_{3} \mathrm{O}_{14}$. The presence of long-range dipolar interactions and intrinsic transverse field further enriches the problem compared to quantum kagome Ising models studied previously $[11,35,36,46,48]$. Our ED calculations on small clusters suggest that the transverse field generates ring-flip tunneling processes that connect degenerate CSF configurations. However, a very recent QMC study on a closely related model shows that this is not sufficient to destroy the low-temperature $\sqrt{3} \times \sqrt{3}$ magnetic order, which is seen over a wide range of $h_{x} / D$ [49]. Understanding if quantum correlations are present at intermediate temperatures, investigating a possible quantum phase transition with increasing transverse field, and elucidating the role of subleading interactions relevant for real compounds appear as promising directions for future research.

Finally, our results highlight the important role hyperfine interactions can play in frustrated quantum magnets based 
on rare-earth ions. It is usually assumed that nuclear spins have a spectator role on the behavior of electronic spins, except in the case of singlet electronic ground states where the hyperfine interaction may induce a cooperative ordering of the combined electronic and nuclear spin system $[80,94,95]$. Our results provide a more subtle example compared to this simple picture due to the highly frustrated nature of spin interactions in $\mathrm{Ho}_{3} \mathrm{Mg}_{2} \mathrm{Sb}_{3} \mathrm{O}_{14}$. Here, hyperfine interactions induce static moments at the single-ion level, and enhance classical correlations at the expense of possible quantum correlations. Yet, due to the frustration, this does not drive the system toward a conventional lowtemperature state with complete long-range magnetic order, but instead to a spin-fragmented state that exhibits predominantly single-ion quantum fluctuations. Finally, since all the stable isotopes of trivalent non-Kramers rare-earth ions have nonzero nuclear spin quantum number, hyperfine interactions have significant effects in other frustrated nonKramers magnets, as for the spin-ice compound $\mathrm{Ho}_{2} \mathrm{Ti}_{2} \mathrm{O}_{7}$ [96], and quantum spin-ice candidates $\operatorname{Pr}_{2} \mathrm{Zr}_{2} \mathrm{O}_{7}$ [22,97] and $\mathrm{Pr}_{2} \mathrm{Hf}_{2} \mathrm{O}_{7}$ [24].

The Department of Energy will provide public access to these results of federally sponsored research in accordance with the DOE Public Access Plan [98].

\section{ACKNOWLEDGMENTS}

We would like to thank Cristian Batista, Owen Benton, Gia-wei Chern, Yuan Wan, Claudio Castelnovo, Laurent Chapon, Radu Coldea, Siân Dutton, Michel Gingras, James Hamp, Peter Holdsworth, Ludovic Jaubert, Gunnar Möller, Jeffrey Rau, and Han Yan for helpful discussions, and Allen Scheie for critical reading of the manuscript. We are indebted to Art Ramirez for providing heat-capacity measurements on our SS sample. The work of Z. D, X. B., J. A. M. P., E. H., and M. M. at Georgia Tech (all analysis, modeling, and interpretation work and all synthesis and measurements on SG samples) was supported by the U.S. Department of Energy, Office of Science, Office of Basic Energy Sciences Neutron Scattering Program under Award No. DE-SC0018660. The work of Z.D and H.Z. at the University of Tennessee (all synthesis and measurements on SS samples) was supported by the National Science Foundation through Grant No. DMR-2003117. H.Z acknowledges support from the NHMFL Visiting Scientist Program, which is supported by NSF Cooperative Agreement No. DMR-1157490 and the State of Florida. J. A. M. P. acknowledges support from Churchill College, Cambridge (neutron data reduction), and the U.S. Department of Energy, Office of Science, Basic Energy Sciences, Materials Sciences and Engineering Division (manuscript co-writing). The research at ISIS Neutron and Muon Source was supported by a beam-time allocation from the STFC (U.K.). The research at Oak Ridge National Laboratory's Spallation Neutron Source and High Flux Isotope Reactor was sponsored by the U.S. DOE, Office of Basic Energy Sciences, Scientific User Facilities Division. This work has been partially supported by the U.S. Department of Energy. ORNL is managed by UTBattelle, LLC, under Contract No. DE-AC05-00OR22725 for the U.S. Department of Energy. The U.S. Government retains and the publisher, by accepting the article for publication, acknowledges that the U.S. Government retains a nonexclusive, paid-up, irrevocable, worldwide license to publish or reproduce the published form of this manuscript, or allow others to do so, for U.S. Government purposes.

Z. D. and X. B. contributed equally to this work.

\section{APPENDIX A: STRUCTURAL AND MAGNETIC MODELS}

The structural model of the SS sample of $\mathrm{Ho}_{3} \mathrm{Mg}_{2} \mathrm{Sb}_{3} \mathrm{O}_{14}$ was obtained by Rietveld corefinements to $50 \mathrm{~K}$ neutron-diffraction data collected using the
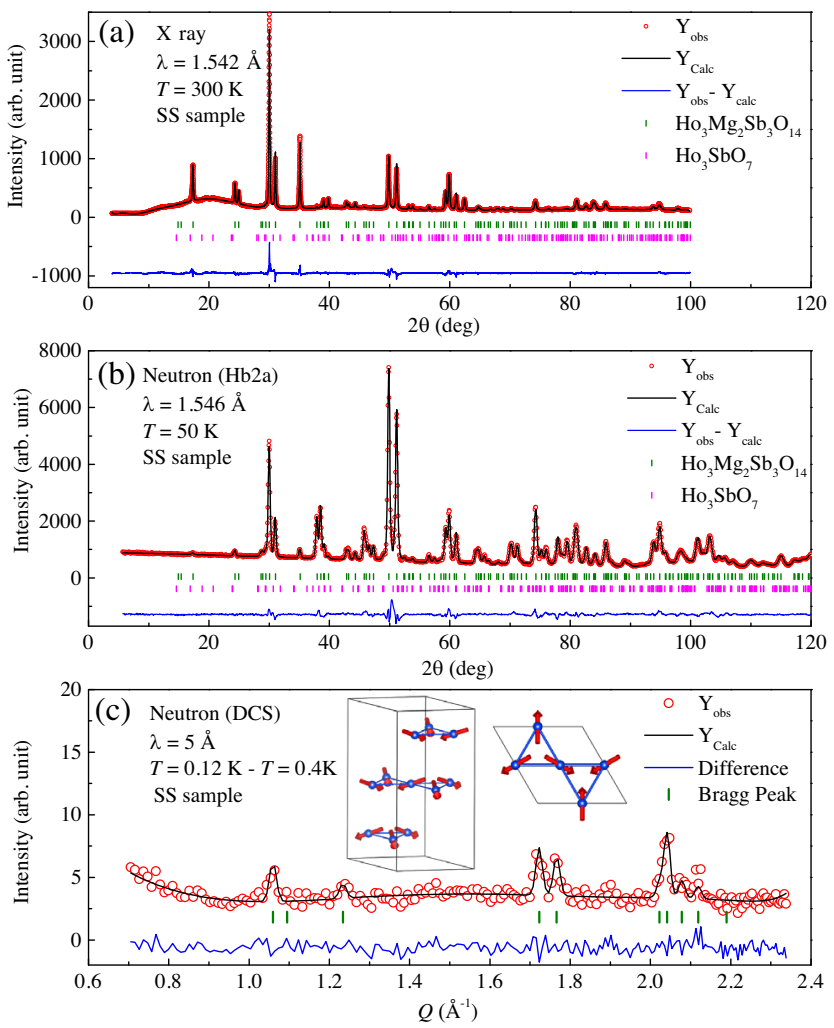

FIG. 7. Rietveld refinements to diffraction data for the SS sample. Corefinements of the crystal structure to neutron and $\mathrm{x}$-ray diffraction data are shown in (a) and (b), respectively. No obvious difference was observed between the x-ray diffraction pattern of SG and SS samples (not shown here). Refinement of the average magnetic structure to low-temperature magnetic diffraction data is shown in (c). In all panels, experimental data are shown as red circles, Rietveld fits as black lines, and difference (data-fit) as blue lines. Inset: illustrations of the all in, all out average magnetic structure within a unit cell and a single kagome layer. 
TABLE I. Crystallographic parameters from Rietveld corefinement to neutron and x-ray diffraction data of SS sample. Anisotropic atomic displacement parameters were used for $\mathrm{Mg} 1$. Fixed parameters are denoted by an asterisk (*). Selected bond lengths and angles are listed.

\begin{tabular}{|c|c|c|c|c|c|}
\hline Atom & Site & $x$ & $y$ & $z$ & Occupancy \\
\hline $\operatorname{Mg} 1$ & $3 a$ & 0 & 0 & 0 & 1 \\
\hline $\mathrm{Mg} 2$ & $3 b$ & 0 & 0 & 0.5 & $0.905(7)$ \\
\hline $\mathrm{Ho}(\mathrm{SD})$ & $3 b$ & 0 & 0 & 0.5 & $0.095(7)$ \\
\hline Но & $9 d$ & 0.5 & 0 & 0.5 & $0.968(2)$ \\
\hline $\operatorname{Mg}(\mathrm{SD})$ & $9 d$ & 0.5 & 0 & 0.5 & $0.032(2)$ \\
\hline $\mathrm{Sb}$ & $9 \mathrm{e}$ & 0.5 & 0 & 0 & 1 \\
\hline $\mathrm{O} 1$ & $6 c$ & 0 & 0 & $0.1166(4)$ & 1 \\
\hline $\mathrm{O} 2$ & $18 \mathrm{~h}$ & $0.5214(2)$ & $0.4786(2)$ & $0.88960(14)$ & 1 \\
\hline $\mathrm{O} 3$ & $18 \mathrm{~h}$ & $0.4694(2)$ & $0.5306(2)$ & $0.35556(13)$ & 1 \\
\hline Lattice parameter $(\AA)$ & \multicolumn{4}{|c|}{ Neutron diffraction, $T=50 \mathrm{~K}$} & \\
\hline$B_{\text {an }}(\operatorname{Mg} 1)\left(\AA^{2}\right)$ & \multicolumn{5}{|c|}{$B_{11}=B_{22}=0.0124(22)$} \\
\hline$B_{\text {iso }}\left(\AA^{2}\right)$ & \multicolumn{4}{|c|}{$\begin{aligned} B(\mathrm{Mg} 2) & =0.07(13), B(\mathrm{O} 1)=0.14(8) \\
B(\mathrm{O} 2) & =0.11(5), B(\mathrm{O} 3)=0.24(5)\end{aligned}$} & \\
\hline \multirow{4}{*}{$\begin{array}{l}\text { Impurity fraction }(\%) \\
\text { Bond lengths }(\AA)\end{array}$} & \multicolumn{5}{|c|}{$f\left(\mathrm{Ho}_{3} \mathrm{SbO}_{7}\right)=2.29(18)$} \\
\hline & \multicolumn{5}{|c|}{$\mathrm{Ho}-\mathrm{O} 1=2.278(3)$} \\
\hline & \multicolumn{5}{|c|}{$\mathrm{Ho}-\mathrm{O} 2=2.456(2)$} \\
\hline & \multicolumn{5}{|c|}{$\mathrm{Ho}-\mathrm{O} 3=2.522(3)$} \\
\hline \multirow[t]{2}{*}{ Bond angles $\left({ }^{\circ}\right)$} & \multirow{2}{*}{\multicolumn{5}{|c|}{$\begin{array}{l}\mathrm{O} 1-\mathrm{Ho}-\mathrm{O} 2=78.69(10) \\
\mathrm{O} 1-\mathrm{Ho}-\mathrm{O} 3=76.54(17)\end{array}$}} \\
\hline & & & & & \\
\hline & \multirow{2}{*}{\multicolumn{5}{|c|}{$\begin{array}{c}\text { X-ray diffraction, } T=300 \mathrm{~K} \\
a=b=7.30939(13), c=17.2696(3)\end{array}$}} \\
\hline Lattice parameter $(\AA)$ & \multicolumn{4}{|c|}{$a=b=7.30939(13), c=17.2696(3)$} & \\
\hline$B_{\text {iso }}\left(\AA^{2}\right)$ & \multicolumn{5}{|c|}{ Overall $B=1.38(3)$} \\
\hline Impurity fraction $(\%)$ & \multicolumn{5}{|c|}{$f\left(\mathrm{Ho}_{3} \mathrm{SbO}_{7}\right)=0.75(11)$} \\
\hline Bond lengths $(\AA)$ & \multicolumn{5}{|c|}{$\mathrm{Ho}-\mathrm{O} 1=2.280(3)$} \\
\hline & \multicolumn{5}{|c|}{$\mathrm{Ho}-\mathrm{O} 2=2.458(2)$} \\
\hline & \multicolumn{5}{|c|}{$\mathrm{Ho}-\mathrm{O} 3=2.524(3)$} \\
\hline \multirow[t]{2}{*}{ Bond angles $\left({ }^{\circ}\right)$} & \multicolumn{5}{|c|}{$\begin{array}{l}\mathrm{O} 1-\mathrm{Ho}-\mathrm{O} 2=78.68(10) \\
\mathrm{O} 1-\mathrm{Ho}-\mathrm{O} 2=76.55(17)\end{array}$} \\
\hline & \multicolumn{5}{|c|}{$\mathrm{O} 1-\mathrm{Ho}-\mathrm{O} 2=76.55(17)$} \\
\hline
\end{tabular}

HB-2A diffractometer [Fig. 7(a)] and $300 \mathrm{~K}$ laboratory x-ray diffraction data [Fig. 7(b)]. Refined values of structural parameters, and selected bond lengths and angles, are given in Table I. The canting angle of the Ising axes with respect to the kagome plane is $22.28(2)^{\circ}$ from the corefinement.

The average magnetic structure at low temperature was obtained by Rietveld refinement to energyintegrated neutron-scattering data collected on the DCS spectrometer on our SS sample [Fig. 7(c)]. We isolated the magnetic Bragg scattering below $T^{*}$ by taking the difference between data measured at 0.12 and $0.4 \mathrm{~K}$. The average AIAO magnetic structure belongs to the same irreducible representation as in $\mathrm{Dy}_{3} \mathrm{Mg}_{2} \mathrm{Sb}_{3} \mathrm{O}_{14}$, described by $\Gamma_{3}$ in Kovalev's notation [99], which is consistent with a spin-fragmented state [41]. The refined ordered moment is $1.70(3) \mu_{B}$ per $\mathrm{Ho}^{3+}$ with a spin canting angle of $24.9^{\circ}$ with respect to the kagome plane.

\section{APPENDIX B: POINT-CHARGE CALCULATIONS}

Because of the low point symmetry at the $\mathrm{Ho}^{3+}$ site, as many as 15 Stevens operators are required to describe the crystal-field Hamiltonian of the system [100]. The number of observables from the inelastic neutron scattering measurements is less than the number of unknown parameters, making conventional fitting procedures impracticable. To circumvent this problem, we calculated the crystal-field levels and wave functions from an effective electrostatic model of point charges using the software package SIMPRE [101]. The model considers eight effective oxygen charges surrounding a $\mathrm{Ho}^{3+}$ ion, consistent with Rietveld refinements to the powder-diffraction data (see Appendix A). The model is then adjusted numerically to match the measured crystal-field spectrum [53]. A similar method using a pointcharge model has recently been applied to study the crystalfield spectrum in isostructural tripod-kagome compounds with $\mathrm{Nd}^{3+}$ and $\mathrm{Pr}^{3+}$ ions [102]. For $\mathrm{Ho}_{3} \mathrm{Mg}_{2} \mathrm{Sb}_{3} \mathrm{O}_{14}$, our 
point charge model predicts that the two lowest-energy singlets are separated by $1.74 \mathrm{~K}\left(h^{x}=0.87 \mathrm{~K}\right)$, and their wave functions are given in the total angular momentum basis $\left(J=8, J^{z}=-8, \ldots,+8\right)$ by

$$
\begin{aligned}
|0\rangle= & 0.690(|8\rangle+|-8\rangle)-0.006(|7\rangle-|-7\rangle) \\
& -0.006(|6\rangle+|-6\rangle)-0.118(|5\rangle-|-5\rangle) \\
& +0.053(|4\rangle+|-4\rangle)+0.019(|3\rangle-|-3\rangle) \\
& +0.003(|2\rangle+|-2\rangle)+0.068(|1\rangle-|-1\rangle)-0.025|0\rangle, \\
|1\rangle= & 0.693(|8\rangle-|-8\rangle)-0.017(|7\rangle+|-7\rangle) \\
& +0.002(|6\rangle-|-6\rangle)-0.106(|5\rangle+|-5\rangle) \\
& -0.008(|4\rangle-|-4\rangle)-0.065(|3\rangle+|-3\rangle) \\
& +0.003(|2\rangle-|-2\rangle)-0.048(|1\rangle-|-1\rangle)-0.000|0\rangle .
\end{aligned}
$$

The matrix element $\alpha=\left\langle 0\left|\hat{J}^{z}\right| 1\right\rangle=7.79$ gives rise to a total magnetic moment of magnitude $9.74 \mu_{B}$ according to Eq. (4).

\section{APPENDIX C: ISOTHERMAL MAGNETIZATION}

To validate our effective Hamiltonian [Eq. (7)], we calculate the isothermal magnetization for $\mathrm{Ho}_{3} \mathrm{Mg}_{2} \mathrm{Sb}_{3} \mathrm{O}_{14}$ and compare it to the experiments. Under a longitudinal magnetic field $H$, the effective single-ion Hamiltonian reads

$$
\mathcal{H}=h^{x} \sigma_{i}^{x}+A_{\mathrm{hf}} I^{z} \sigma^{z}+\mu_{0} \mu_{B} \alpha g_{J} H \sigma^{z} .
$$

With eigenvalues $E_{i}$ and eigenvectors $|i\rangle$ diagonalized from the above Hamiltonian, the magnetization along the local Ising axis is

$$
M^{z}(H, T)=\alpha g_{J} \sum_{i} e^{-E_{i} / k_{B} T}\left\langle i\left|\sigma^{z}\right| i\right\rangle / \sum_{i} e^{-E_{i} / k_{B} T} .
$$

Along with a Van Vleck paramagnetic term $\chi_{\mathrm{VV}}$, the powderaveraged magnetization is

$$
M_{\text {powder }}(H, T)=\int_{0}^{\pi} M^{z}(H, T) \sin \theta d \theta+\chi_{\mathrm{Vv}} H .
$$

With $\alpha=7.79$ determined in Appendix B, $h_{x}=$ $0.85 \mathrm{~K}, A_{\mathrm{hf}}=0.319 \mathrm{~K}$, and a fitted value of $\chi_{\mathrm{Vv}} / \mu_{0}=$ $0.0148 \mu_{B} / T$, the calculated $M_{\text {powder }}(H, T)$ agrees well with the measured isothermal magnetization in the paramagnetic regime between 1.8 and $40 \mathrm{~K}$ (Fig. 8). At low temperatures, such as between 5 and $1.8 \mathrm{~K}$, deviations from the calculation become appreciable due to the development of spin-spin correlations that are not included in this model.

\section{APPENDIX D: PARAMAGNETIC EFFECTIVE-FIELD FITS}

We used an effective-field approach to calculate the inelastic neutron-scattering pattern in the paramagnetic phase, based on the Onsager reaction-field approximation 88]]. Full details of this method are given in Ref. [87].

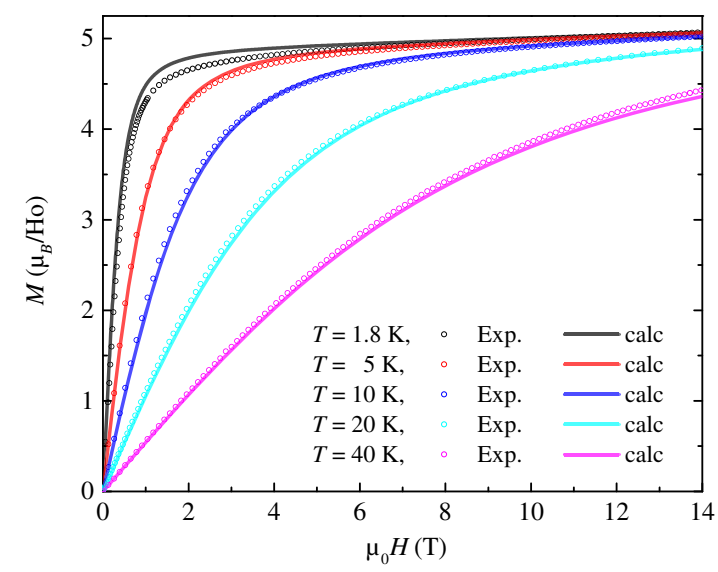

FIG. 8. Isothermal magnetization measurements (open symbols) on the $\mathrm{SG}$ sample of $\mathrm{Ho}_{3} \mathrm{Mg}_{2} \mathrm{Sb}_{3} \mathrm{O}_{14}$ at various temperatures. Solid lines were calculated according to Eq. (C3) with fixed values of $\alpha=7.79, h_{x}=0.85 \mathrm{~K}, A_{\mathrm{hf}}=0.319 \mathrm{~K}$, and a fitted Van Vleck term $\chi_{\mathrm{Vv}} / \mu_{0}=0.0148 \mu_{B} / T$.

In this approximation, the inelastic scattering function is given by

$S(\mathbf{Q}, \omega)=\frac{1}{n \pi\left[1-e^{-\omega / T}\right]} \sum_{\mu=1}^{N}\left|\mathbf{F}_{\mu}^{\perp}(\mathbf{Q})\right|^{2} \operatorname{Im}\left[\chi_{\mu}(\mathbf{Q}, \omega)\right]$,

where $n=3$ is the number of $\mathrm{Ho}^{3+}$ ions in the primitive unit cell, $\omega$ is energy transfer in $\mathrm{K}$, and the susceptibility for each normal mode $\mu$ is given in Eq. (11) $[84,103]$. The magnetic structure factor is given by

$$
\mathbf{F}_{\mu}^{\perp}(\mathbf{Q})=\sum_{i=1}^{N} \mathbf{z}_{i}^{\perp} U_{i \mu}(\mathbf{Q}) \exp \left(i \mathbf{Q} \cdot \mathbf{r}_{i}\right),
$$

where $\mathbf{Q}$ is the scattering vector, $\mathbf{r}_{i}$ is the position of magnetic ion $i$ in the primitive cell, and $\mathbf{z}_{i}^{\perp}$ is its local Ising axis projected perpendicular to $\mathbf{Q}$. The eigenvectors $U_{i \mu}$ and mode energies $\lambda_{\mu}$ are given at each $\mathbf{Q}$ as the solutions of

$$
\lambda_{\mu}(\mathbf{Q}) U_{i \mu}(\mathbf{Q})=\sum_{j} J_{i j}(\mathbf{Q}) U_{j \mu}(\mathbf{Q}),
$$

where the Fourier-transformed interaction $J_{i j}(\mathbf{Q})=$ $\sum_{\mathbf{R}} J_{i j}(\mathbf{R}) \exp (i \mathbf{Q} \cdot \mathbf{R})$ includes nearest-neighbor exchange and long-range dipolar contributions, and $\mathbf{R}$ is the lattice vector connecting atoms $i$ and $j$. The dipolar interaction was calculated using Ewald summation [89]. The Onsager reaction field $\lambda$ is determined by enforcing the totalmoment sum rule:

$$
\frac{1}{n N_{\mathbf{q}}} \sum_{i, \mathbf{q}}\left\langle\sigma_{i}^{z}(\mathbf{q}) \sigma_{i}^{z}(-\mathbf{q})\right\rangle=1 .
$$

The scattering intensities were calculated as

$$
I(\omega)=C\left[\frac{\mu f(Q)}{\mu_{B}}\right]^{2} \int_{Q_{0}}^{Q_{1}}\langle S(\mathbf{Q}, \omega)\rangle_{Q} d Q,
$$


where $Q_{0}=0.4 \AA^{-1}$ and $Q_{1}=1.6 \AA^{-1}$, and

$$
I(Q)=C\left[\frac{\mu f(Q)}{\mu_{B}}\right]^{2} \int_{-\omega^{\prime}}^{\omega^{\prime}}\langle S(\mathbf{Q}, \omega)\rangle_{\omega} d \omega
$$

where $\omega^{\prime}=20 \mathrm{~K}$, angle brackets here denote numerical spherical averaging, $f(Q)$ is the $\mathrm{Ho}^{3+}$ magnetic form factor [104], $\mu=10 \mu_{B}$ is the total magnetic moment per $\mathrm{Ho}^{3+}$, and the constant $C=\left(\gamma_{n} r_{e} / 2\right)^{2}=0.07265 \mathrm{~b}$. The integrals were performed numerically.

\section{APPENDIX E: EXACT DIAGONALIZATION}

Because of computational limitations, our ED calculations were restricted to one tripod-kagome layer. Cluster sizes of $N=12$ and 18 with different shapes were studied under periodic boundary conditions. Long-range dipolar interactions were summed over periodic copies of the cluster cells up to a distance of $500 r_{\mathrm{NN}}$.

The existence of a quantum phase transition at small $h^{x} / D$ as well as the $\left(h^{x} / D\right)^{6}$ dependence of the exciton energy are independent of size or shape of the clusters (Fig. 9). When truncating the dipolar interaction at the first nearest neighbor, our dipolar TIM becomes an exchange TIM model that has been investigated previously $[46,48]$. Our results are consistent with these studies for which the ground states obtained at low field are continuously connected to the high-field paramagnetic state with a $h^{x} / D$ dependence of the exciton energy.

(a)
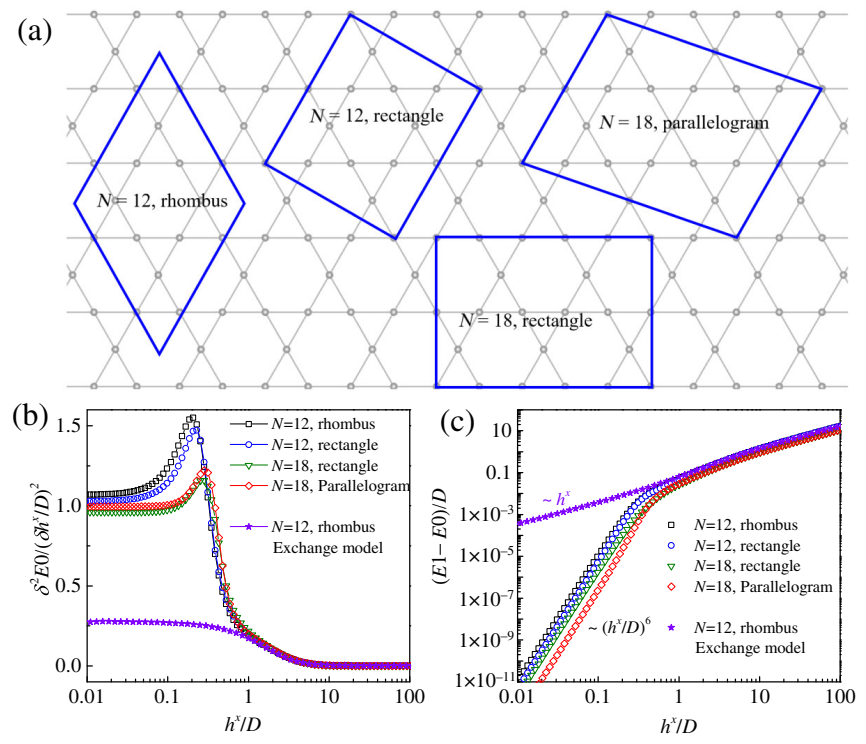

FIG. 9. (a) Different clusters used for the exact diagonalization (ED) calculations of size $N=12,18$. ED results of Eq. (10) with $J_{\mathrm{NN}}=0$, and $A_{\mathrm{hf}}=0$, showing (b) second derivative of the ground-state energy, and (c) energy gap between the ground state and first excited state. "Exchange model" denotes a transverse Ising model with nearest-neighbor exchange couplings only.
When considering the hyperfine interactions, Eq. (10) can be put into a block-diagonal form because $I^{z}$ remains a good quantum number. Then each block has a set of fixed nuclear spin numbers $\left\{I_{1}^{z}, I_{2}^{z}, \ldots, I_{N}^{z}\right\}$ and the hyperfine term is treated as a site-dependent local longitudinal field. Therefore, we find the global ground state by diagonalizing the Hamiltonian in each of the $8^{N}$ nuclear spin blocks independently. As expected, the ground states of the Hamiltonian live in the nuclear spin blocks where $I_{i}^{z}=$ $\pm 7 / 2$ for every site. The nuclear spin configurations of these ground states match with those electronic spins of the CSF states and thus possesses the same degeneracy. In other words, the degeneracy of a classical Ising system is restored in a quantum system through the nuclear spin channel. Recall that the ground state in the absence of hyperfine interaction is a superposition of all CSF basis states. The key effect of the hyperfine interaction is to promote the probability weight of one of the basis states over the others, which means it is suppressing quantum effects and driving the system to the classical limit, as discussed in the main text.

\section{APPENDIX F: MEAN-FIELD MONTE CARLO SIMULATIONS}

The key idea of our mean-field Monte Carlo simulation is to generalize the local states from the eigenstates of $\sigma^{z}$ to those of the single-ion mean-field Hamiltonian. These two sets of eigenstates are identical at the limit of $h^{x}=0$. The primary effect of the transverse field in our current calculation is to introduce quantum fluctuation at the level of a single site.

We keep track of the expectation values of $\sigma_{i}^{z}, \sigma_{i}^{x}$, and $I_{i}^{z}$ at each site during the simulation. The energy of the

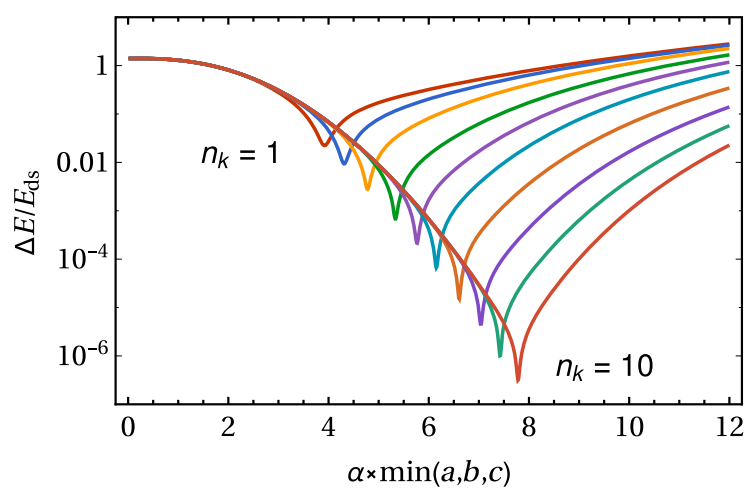

FIG. 10. The relative difference of total energy between the Ewald summation and direct summation as a function of the splitting parameter $\alpha$ and reciprocal space cutoff $n_{k}$. The real space cutoff is taken to be $r_{c}=\min (a, b, c) / 2$, where $a, b$, and $c$ are the dimension of $5 \times 3 \times 2$ orthorhombic supercell. The realspace cutoff in the direct sum is $r_{c}=1000 r_{\mathrm{NN}}$. The calculation is averaged over 50 random spin configurations. We take the reciprocal-space cutoff $n_{k}=10$ and the best splitting parameter $\alpha=7.78 / \min (a, b, c)$. The same procedure is performed to find the best splitting parameter $\alpha=8.10 / \min (a, b, c)$ for the $10 \times$ $6 \times 4$ simulation box with the same reciprocal-space cutoff. 

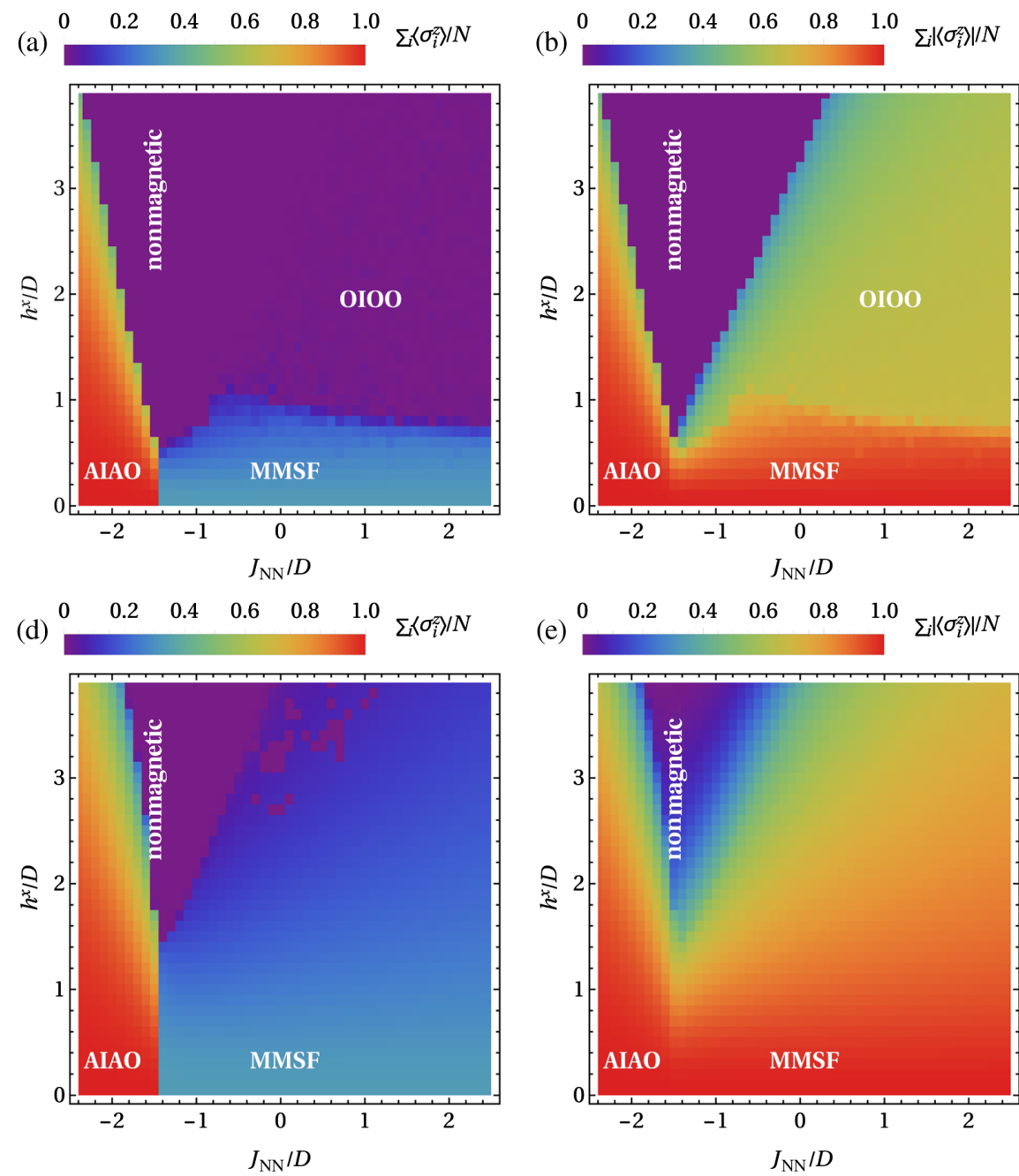
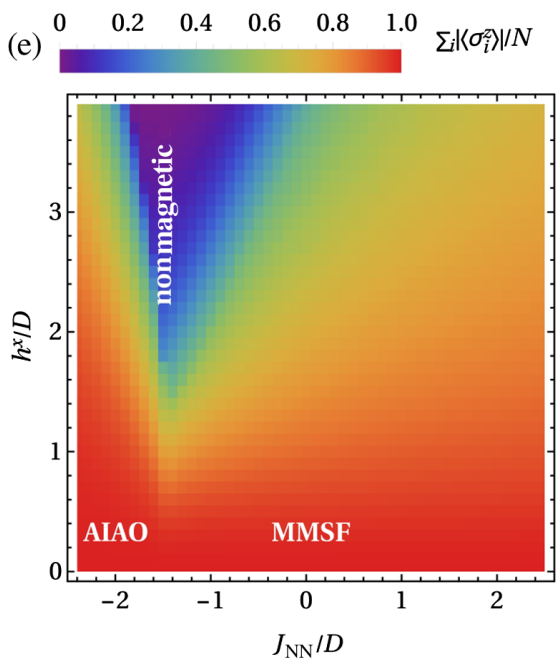
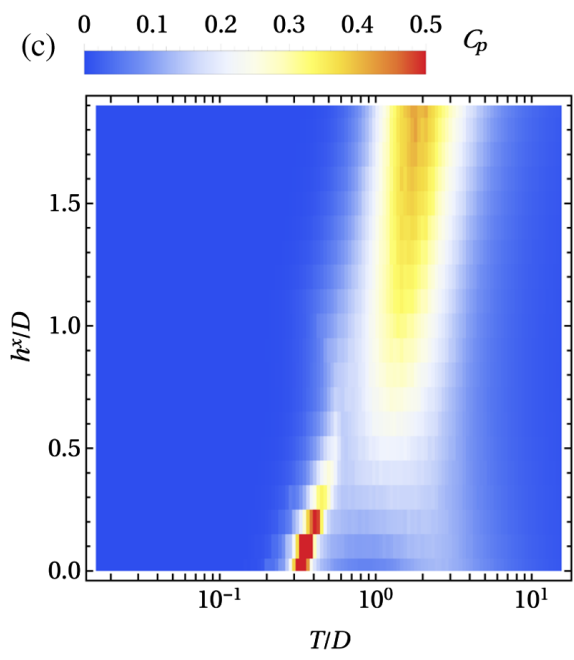

(f) $\begin{array}{lllllllll} & 0 & 0.25 & 0.50 & 0.75 & 1.00 & 1.25 & 1.50 & C_{p}\end{array}$

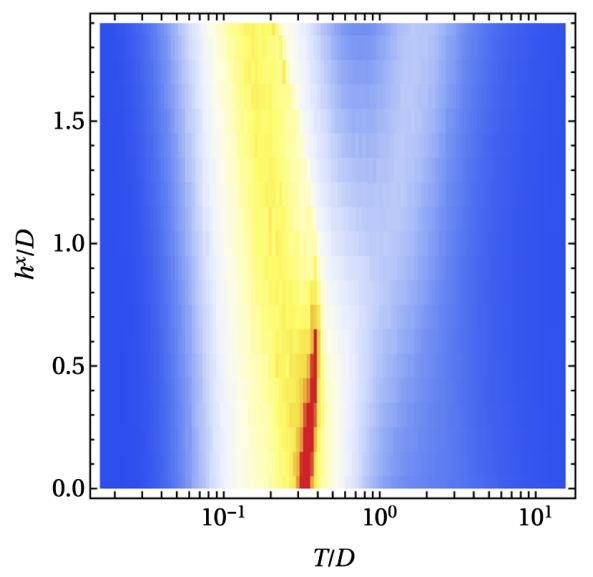

FIG. 11. Comparison of the ordered moment (a),(d), the static moment (b),(e), and the specific heat (c),(f) without the hyperfine coupling (top three panels) and with the hyperfine coupling $A_{\mathrm{hf}}=0.319 \mathrm{~K}$ (bottom three panels) from our MFMC simulations. The dipolar interaction strength is $1.29 \mathrm{~K}$. The temperature of (a), (b), (d), and (e) is $0.12 \mathrm{~K}$. The exchange interaction strength in (c) and (f) is $-0.64 \mathrm{~K}$. The OIOO phase is destroyed by including the hyperfine coupling as shown by comparing (a) and (d).

system is computed by replacing the spin operators in the Hamiltonian Eq. (10) by their corresponding expectation values. The updates of static spin $\left\langle\sigma_{i}^{z}\right\rangle$ described in the main text are accompanied by corresponding changes in $\left\langle\sigma_{i}^{x}\right\rangle$; e.g., $\left\langle\sigma_{i}^{x}\right\rangle$ becomes $-\left\langle\sigma_{i}^{x}\right\rangle$ if the static spin is flipped. When a new mean-field state is proposed, we update both $\left\langle\sigma_{i}^{x}\right\rangle$ and $\left\langle\sigma_{i}^{z}\right\rangle$.

We used an orthorhombic $5 \times 3 \times 2$ supercell of the crystallographic unit cell (see Appendix A) containing $N=540$ sites in six kagome layers to obtain the heat capacity and the phase diagram shown in Figs. 3 and 6, respectively. A $10 \times 6 \times 4$ simulation box containing $N=4320$ sites was used to compute the Fourier transform of the static spin correlation function shown in Fig. 6(c) and the powder neutron-scattering patterns shown in Fig. 4. The long-range dipolar interaction was treated by Ewald summation with tinfoil boundary condition at infinity $[105,106]$. The interaction matrix was computed only once at the beginning of the simulation using the formulas for noncubic unit cells given in Ref. [107]. Suitable Ewald parameters were chosen by comparing with the result from direct summmation (Fig. 10). The canted local $\mathbf{z}$ axis of $22.28^{\circ}$ obtained from the structure refinement was implemented. A simulated annealing process was performed during simulations. We started with random spin configurations at $20 \mathrm{~K}$ and cooled the system to $0.1 \mathrm{~K}$ with an exponential rate of 0.95 . At each temperature, we performed 10000 equilibration sweeps, then collected samples after every 30 sweeps. The heat capacity shown in Fig. 3 and 11 was averaged over 30000 samples. One sweep consisted of $N$ MC steps, where $N$ is the number of sites in the simulation box.

The calculated magnetic scattering shown in Fig. 4(b) was obtained as the sum of static diffuse $I_{\text {diff }}(Q)$, Bragg $I_{\text {Bragg }}(Q)$, and inelastic $I_{\text {inelastic }}(Q)$ contributions, minus the high-temperature paramagnetic $I_{\text {para }}(Q)$ contribution, 


$$
I_{\text {sub }}(Q)=I_{\text {diff }}(Q)+I_{\text {Bragg }}(Q)+I_{\text {inelastic }}(Q)-I_{\text {para }}(Q),
$$

where the Bragg and diffuse contributions are calculated following Ref. [41]. The inelastic contribution was given by

$$
I_{\text {inelastic }}(Q)=\frac{2}{3} C\left[\frac{\mu f(Q)}{\mu_{B}}\right]^{2} \frac{1}{N} \sum_{i}\left(1-\left|\left\langle\sigma_{i}^{z}\right\rangle\right|^{2}\right) .
$$

The $Q$-averaged inelastic spectrum $I(\omega)$ was calculated as

$$
I(\omega)=\frac{2}{3} C\left[\frac{\mu}{\mu_{B}}\right]^{2} \frac{1}{N} \sum_{i}\left[W_{i} \delta(\omega)+\left(1-W_{i}\right) \delta\left(\omega-E_{i}\right)\right],
$$

where $E_{i}=2 \sqrt{\left(h_{i}^{x}\right)^{2}+\left(h_{i}^{z}\right)^{2}}$ is the energy of the singlespin excitation and $W_{i}=\left(h_{i}^{z}\right)^{2} /\left[\left(h_{i}^{x}\right)^{2}+\left(h_{i}^{z}\right)^{2}\right]$ is the elastic spectral weight. The green line shown in Fig. 4(c) was obtained by convoluting $I(\omega)$ with the experimental energy resolution at the elastic line ( $\mathrm{FWHM} \approx 1 \mathrm{~K})$.

[1] L. Balents, Spin Liquids in Frustrated Magnets, Nature (London) 464, 199 (2010).

[2] M. J. Harris, S. T. Bramwell, D. F. McMorrow, T. Zeiske, and K.W. Godfrey, Geometrical Frustration in the Ferromagnetic Pyrochlore $\mathrm{Ho}_{2} \mathrm{Ti}_{2} \mathrm{O}_{7}$, Phys. Rev. Lett. 79, 2554 (1997).

[3] S. T. Bramwell and M. J. P. Gingras, Spin Ice State in Frustrated Magnetic Pyrochlore Materials, Science 294, 1495 (2001).

[4] C. Castelnovo, R. Moessner, and S. L. Sondhi, Magnetic Monopoles in Spin Ice, Nature (London) 451, 42 (2008).

[5] D. J. P. Morris, D. A. Tennant, S. A. Grigera, B. Klemke, C. Castelnovo, R. Moessner, C. Czternasty, M. Meissner, K. C. Rule, J.-U. Hoffmann, K. Kiefer, S. Gerischer, D. Slobinsky, and R. S. Perry, Dirac Strings and Magnetic Monopoles in the Spin Ice $\mathrm{Dy}_{2} \mathrm{Ti}_{2} \mathrm{O}_{7}$, Science 326, 411 (2009).

[6] H. Kadowaki, N. Doi, Y. Aoki, Y. Tabata, T. J. Sato, J. W. Lynn, K. Matsuhira, and Z. Hiroi, Observation of Magnetic Monopoles in Spin Ice, J. Phys. Soc. Jpn. 78, 103706 (2009).

[7] T. Fennell, P. P. Deen, A. R. Wildes, K. Schmalzl, D. Prabhakaran, A. T. Boothroyd, R. J. Aldus, D. F. McMorrow, and S. T. Bramwell, Magnetic Coulomb Phase in the Spin Ice $\mathrm{Ho}_{2} \mathrm{Ti}_{2} \mathrm{O}_{7}$, Science 326, 415 (2009).

[8] M. Hermele, M. P. A. Fisher, and L. Balents, Pyrochlore Photons: The U(1) Spin Liquid in a $S=\frac{1}{2}$ ThreeDimensional Frustrated Magnet, Phys. Rev. B 69, 064404 (2004).

[9] L. Savary and L. Balents, Coulombic Quantum Liquids in Spin-1/2 Pyrochlores, Phys. Rev. Lett. 108, 037202 (2012).
[10] M. J. P. Gingras and P. A. McClarty, Quantum Spin Ice: A Search for Gapless Quantum Spin Liquids in Pyrochlore Magnets, Rep. Prog. Phys. 77, 056501 (2014).

[11] R. Moessner, S. L. Sondhi, and P. Chandra, TwoDimensional Periodic Frustrated Ising Models in a Transverse Field, Phys. Rev. Lett. 84, 4457 (2000).

[12] L.-P. Henry and T. Roscilde, Order-by-Disorder and Quantum Coulomb Phase in Quantum Square Ice, Phys. Rev. Lett. 113, 027204 (2014).

[13] B. Tomasello, C. Castelnovo, R. Moessner, and J. Quintanilla, Single-Ion Anisotropy and Magnetic Field Response in the Spin-Ice Materials $\mathrm{Ho}_{2} \mathrm{Ti}_{2} \mathrm{O}_{7}$ and $\mathrm{Dy}_{2} \mathrm{Ti}_{2} \mathrm{O}_{7}$, Phys. Rev. B 92, 155120 (2015).

[14] L. Savary and L. Balents, Disorder-Induced Quantum Spin Liquid in Spin Ice Pyrochlores, Phys. Rev. Lett. 118, 087203 (2017).

[15] H. D. Zhou, C. R. Wiebe, J. A. Janik, L. Balicas, Y. J. Yo, Y. Qiu, J. R. D. Copley, and J. S. Gardner, Dynamic Spin Ice: $\operatorname{Pr}_{2} \mathrm{Sn}_{2} \mathbf{O}_{7}$, Phys. Rev. Lett. 101, 227204 (2008).

[16] K. A. Ross, L. Savary, B. D. Gaulin, and L. Balents, Quantum Excitations in Quantum Spin Ice, Phys. Rev. X 1, 021002 (2011).

[17] J. D. Thompson, P. A. McClarty, H. M. Rønnow, L. P. Regnault, A. Sorge, and M. J. P. Gingras, Rods of Neutron Scattering Intensity in $\mathrm{Yb}_{2} \mathrm{Ti}_{2} \mathrm{O}_{7}$ : Compelling Evidence for Significant Anisotropic Exchange in a Magnetic Pyrochlore Oxide, Phys. Rev. Lett. 106, 187202 (2011).

[18] T. Fennell, M. Kenzelmann, B. Roessli, M. K. Haas, and R. J. Cava, Power-Law Spin Correlations in the Pyrochlore Antiferromagnet $\mathrm{Tb}_{2} \mathrm{Ti}_{2} \mathrm{O}_{7}$, Phys. Rev. Lett. 109, 017201 (2012).

[19] R. Sibille, E. Lhotel, V. Pomjakushin, C. Baines, T. Fennell, and M. Kenzelmann, Candidate Quantum Spin Liquid in the $\mathrm{Ce}^{3+}$ Pyrochlore Stannate $\mathrm{Ce}_{2} \mathrm{Sn}_{2} \mathrm{O}_{7}$, Phys. Rev. Lett. 115, 097202 (2015).

[20] R. Sibille, E. Lhotel, M. C. Hatnean, G. Balakrishnan, B. Fåk, N. Gauthier, T. Fennell, and M. Kenzelmann, Candidate Quantum Spin Ice in the Pyrochlore $\operatorname{Pr}_{2} \mathrm{Hf}_{2} \mathrm{O}_{7}$, Phys. Rev. B 94, 024436 (2016).

[21] S. Petit, E. Lhotel, B. Canals, M. C. Hatnean, J. Ollivier, H. Mutka, E. Ressouche, A. R. Wildes, M. R. Lees, and G. Balakrishnan, Observation of Magnetic Fragmentation in Spin Ice, Nat. Phys. 12, 746 (2016).

[22] J.-J. Wen, S. M. Koohpayeh, K. A. Ross, B. A. Trump, T. M. McQueen, K. Kimura, S. Nakatsuji, Y. Qiu, D. M. Pajerowski, J. R. D. Copley, and C. L. Broholm, Disordered Route to the Coulomb Quantum Spin Liquid: Random Transverse Fields on Spin Ice in $\mathrm{Pr}_{2} \mathrm{Zr}_{2} \mathrm{O}_{7}$, Phys. Rev. Lett. 118, 107206 (2017).

[23] E. Lhotel, S. Petit, M. C. Hatnean, J. Ollivier, H. Mutka, E. Ressouche, M. R. Lees, and G. Balakrishnan, Evidence for Dynamic Kagome Ice, Nat. Commun. 9, 3786 (2018).

[24] R. Sibille, N. Gauthier, H. Yan, M. C. Hatnean, J. Ollivier, B. Winn, U. Filges, G. Balakrishnan, M. Kenzelmann, N. Shannon, and T. Fennell, Experimental Signatures of Emergent Quantum Electrodynamics in $\mathrm{Pr}_{2} \mathrm{Hf}_{2} \mathrm{O}_{7}$, Nat. Phys. 14, 711 (2018).

[25] C. Mauws, A. M. Hallas, G. Sala, A. A. Aczel, P. M. Sarte, J. Gaudet, D. Ziat, J. A. Quilliam, J. A. Lussier, M. Bieringer, H. D. Zhou, A. Wildes, M. B. Stone, D. Abernathy, 
G. M. Luke, B. D. Gaulin, and C. R. Wiebe, DipolarOctupolar Ising Antiferromagnetism in $\mathrm{Sm}_{2} \mathrm{Ti}_{2} \mathrm{O}_{7}: A$ Moment Fragmentation Candidate, Phys. Rev. B 98, 100401(R) (2018).

[26] L. D. C. Jaubert, O. Benton, J. G. Rau, J. Oitmaa, R. R. P. Singh, N. Shannon, and M. J. P. Gingras, Are Multiphase Competition and Order by Disorder the Keys to Understanding $\mathrm{Yb}_{2} \mathrm{Ti}_{2} \mathrm{O}_{7}$ ?, Phys. Rev. Lett. 115, 267208 (2015).

[27] H. Yan, O. Benton, L. Jaubert, and N. Shannon, Theory of Multiple-Phase Competition in Pyrochlore Magnets with Anisotropic Exchange with Application to $\mathrm{Yb}_{2} \mathrm{Ti}_{2} \mathrm{O}_{7}, \mathrm{Er}_{2} \mathrm{Ti}_{2} \mathrm{O}_{7}$, and $\mathrm{Er}_{2} \mathrm{Sn}_{2} \mathrm{O}_{7}$, Phys. Rev. B 95, 094422 (2017).

[28] J. D. Thompson, P. A. McClarty, D. Prabhakaran, I. Cabrera, T. Guidi, and R. Coldea, Quasiparticle Breakdown and Spin Hamiltonian of the Frustrated Quantum Pyrochlore $\mathrm{Yb}_{2} \mathrm{Ti}_{2} \mathrm{O}_{7}$ in a Magnetic Field, Phys. Rev. Lett. 119, 057203 (2017).

[29] G. Sala, M. J. Gutmann, D. Prabhakaran, D. Pomaranski, C. Mitchelitis, J. B. Kycia, D. G. Porter, C. Castelnovo, and J. P. Goff, Vacancy Defects and Monopole Dynamics in Oxygen-Deficient Pyrochlores, Nat. Mater. 13, 488 (2014).

[30] N. Martin, P. Bonville, E. Lhotel, S. Guitteny, A. Wildes, C. Decorse, M. Ciomaga Hatnean, G. Balakrishnan, I. Mirebeau, and S. Petit, Disorder and Quantum Spin Ice, Phys. Rev. X 7, 041028 (2017).

[31] A. Mostaed, G. Balakrishnan, M. R. Lees, Y. Yasui, L.-J. Chang, and R. Beanland, Atomic Structure Study of the Pyrochlore $\mathrm{Yb}_{2} \mathrm{Ti}_{2} \mathrm{O}_{7}$ and Its Relationship with LowTemperature Magnetic Order, Phys. Rev. B 95, 094431 (2017).

[32] N. Shannon, O. Sikora, F. Pollmann, K. Penc, and P. Fulde, Quantum Ice: A Quantum Monte Carlo Study, Phys. Rev. Lett. 108, 067204 (2012).

[33] Y. Kato and S. Onoda, Numerical Evidence of Quantum Melting of Spin Ice: Quantum-to-Classical Crossover, Phys. Rev. Lett. 115, 077202 (2015).

[34] A. S. Wills, R. Ballou, and C. Lacroix, Model of Localized Highly Frustrated Ferromagnetism: The Kagomé Spin Ice, Phys. Rev. B 66, 144407 (2002).

[35] J. Carrasquilla, Z. Hao, and R. G. Melko, A TwoDimensional Spin Liquid in Quantum Kagome Ice, Nat. Commun. 6, 7421 (2015).

[36] K.-H. Wu, Y.-P. Huang, and Y.-J. Kao, Tunneling-Induced Restoration of Classical Degeneracy in Quantum Kagome Ice, Phys. Rev. B 99, 134440 (2019).

[37] G. Möller and R. Moessner, Magnetic Multipole Analysis of Kagome and Artificial Spin-Ice Dipolar Arrays, Phys. Rev. B 80, 140409(R) (2009).

[38] G.-W. Chern, P. Mellado, and O. Tchernyshyov, TwoStage Ordering of Spins in Dipolar Spin Ice on the Kagome Lattice, Phys. Rev. Lett. 106, 207202 (2011).

[39] B. Canals, I.-A. Chioar, V.-D. Nguyen, M. Hehn, D. Lacour, F. Montaigne, A. Locatelli, T. O. Mentes, B.S. Burgos, and N. Rougemaille, Fragmentation of Magnetism in Artificial Kagome Dipolar Spin Ice, Nat. Commun. 7, 11446 (2016).
[40] M. E. Brooks-Bartlett, S. T. Banks, L. D. C. Jaubert, A. Harman-Clarke, and P. C. W. Holdsworth, MagneticMoment Fragmentation and Monopole Crystallization, Phys. Rev. X 4, 011007 (2014).

[41] J. A. M. Paddison, H. S. Ong, J. O. Hamp, P. Mukherjee, X. Bai, M. G. Tucker, N. P. Butch, C. Castelnovo, M. Mourigal, and S. E. Dutton, Emergent Order in the Kagome Ising Magnet $\mathrm{Dy}_{3} \mathrm{Mg}_{2} \mathrm{Sb}_{3} \mathrm{O}_{14}$, Nat. Commun. 7, 13842 (2016).

[42] E. Lefrançois, V. Cathelin, E. Lhotel, J. Robert, P. Lejay, C. V. Colin, B. Canals, F. Damay, J. Ollivier, B. Fåk, L. C. Chapon, R. Ballou, and V. Simonet, Fragmentation in Spin Ice from Magnetic Charge Injection, Nat. Commun. 8, 209 (2017).

[43] X. Bai, J. A. M. Paddison, S. E. Dutton, and M. Mourigal (to be published).

[44] M. Stern, C. Castelnovo, R. Moessner, V. Oganesyan, and S. Gopalakrishnan, Quantum Percolation of Monopole Paths and the Response of Quantum Spin Ice, arXiv: 1911.05742.

[45] O. Benton, O. Sikora, and N. Shannon, Seeing the Light: Experimental Signatures of Emergent Electromagnetism in a Quantum Spin Ice, Phys. Rev. B 86, 075154 (2012).

[46] R. Moessner and S. L. Sondhi, Ising Models of Quantum Frustration, Phys. Rev. B 63, 224401 (2001).

[47] G. Misguich, D. Serban, and V. Pasquier, Quantum Dimer Model on the Kagome Lattice: Solvable Dimer-Liquid and Ising Gauge Theory, Phys. Rev. Lett. 89, 137202 (2002).

[48] P. Nikolić and T. Senthil, Theory of the Kagome Lattice Ising Antiferromagnet in Weak Transverse Fields, Phys. Rev. B 71, 024401 (2005).

[49] Y. Wang, S. Humeniuk, and Y. Wan, Tuning the Two-Step Melting of Magnetic Order in a Dipolar Kagome Spin Ice by Quantum Fluctuations, Phys. Rev. B 101, 134414 (2020).

[50] Z. L. Dun, J. Trinh, M. Lee, E. S. Choi, K. Li, Y. F. Hu, Y.X. Wang, N. Blanc, A. P. Ramirez, and H. D. Zhou, Structural and Magnetic Properties of Two Branches of the Tripod-Kagome-Lattice Family $A_{2} R_{3} \mathrm{Sb}_{3} \mathrm{O}_{14}(A=\mathrm{Mg}$, $\mathrm{Zn} ; \mathrm{R}=\mathrm{Pr}, \mathrm{Nd}, \mathrm{Gd}, \mathrm{Tb}, \mathrm{Dy}, \mathrm{Ho}, \mathrm{Er}, \mathrm{Yb})$, Phys. Rev. B 95, 104439 (2017).

[51] Z. L. Dun, J. Trinh, K. Li, M. Lee, K. W. Chen, R. Baumbach, Y. F. Hu, Y.X. Wang, E. S. Choi, B. S. Shastry, A. P. Ramirez, and H. D. Zhou, Magnetic Ground States of the Rare-Earth Tripod Kagome Lattice $\mathrm{Mg}_{2} \mathrm{RE}_{3} \mathrm{Sb}_{3} \mathrm{O}_{14}$ ( $\left.\mathrm{RE}=\mathrm{Gd}, D y, E r\right)$, Phys. Rev. Lett. 116, 157201 (2016).

[52] Y.-Li Wang and B. R. Cooper, Collective Excitations and Magnetic Ordering in Materials with Singlet Crystal-Field Ground State, Phys. Rev. 172, 539 (1968).

[53] Z. L. Dun, X. Bai, M. B. Stone, H. D. Zhou, and M. Mourigal, Effective Point-Charge Analysis of Crystal Electric Fields-Application to Rare-Earth Pyrochlores and Tripod Kagome Magnets $R_{3} \mathrm{Mg}_{2} \mathrm{Sb}_{3} \mathrm{O}_{14}$, arXiv: 2004.10957.

[54] T. Fennell, S. T. Bramwell, and M. A. Green, Structural and Magnetic Characterization of $\mathrm{Ho}_{3} \mathrm{SbO}_{7}$ and $\mathrm{Dy}_{3} \mathrm{SbO}_{7}$, Can. J. Phys. 79, 1415 (2001). 
[55] V. O. Garlea, B. C. Chakoumakos, S. A. Moore, G. B. Taylor, T. Chae, R. G. Maples, R. A. Riedel, G. W. Lynn, and D. L. Selby, The High-Resolution Powder Diffractometer at the High Flux Isotope Reactor, Appl. Phys. A 99, 531 (2010).

[56] J. Rodríguez-Carvajal, Recent Advances in Magnetic Structure Determination by Neutron Powder Diffraction, Physica (Amsterdam) 192B, 55 (1993).

[57] G. E. Granroth, A. I. Kolesnikov, T. E. Sherline, J. P. Clancy, K. A. Ross, J. P. C. Ruff, B. D. Gaulin, and S. E. Nagler, SEQUOIA: A Newly Operating Chopper Spectrometer at the SNS, J. Phys. Conf. Ser. 251, 012058 (2010).

[58] J. R. D. Copley and J. C. Cook, The Disk Chopper Spectrometer at NIST: A New Instrument for Quasielastic Neutron Scattering Studies, Special issue on Quasielastic Neutron Scattering of Structural Dynamics in Condensed Matter, edited by R. E. Lechner, Chem. Phys. 292, 477 (2003).

[59] R. T. Azuah, L. R. Kneller, Y. Qiu, P. L. W. TregennaPiggott, C. M. Brown, J. R. D. Copley, and R. M. Dimeo, DAVE: A Comprehensive Software Suite for the Reduction, Visualization, and Analysis of Low Energy Neutron Spectroscopic Data, J. Res. Natl. Inst. Stand. Technol. 114, 341 (2009).

[60] J. A. K. Howard, O. Johnson, A. J. Schultz, and A. M. Stringer, Determination of the Neutron Absorption Cross Section for Hydrogen as a Function of Wavelength with a Pulsed Neutron Source, J. Appl. Crystallogr. 20, 120 (1987).

[61] S. Rosenkranz, A. P. Ramirez, A. Hayashi, R. J. Cava, R. Siddharthan, and B. S. Shastry, Crystal-Field Interaction in the Pyrochlore Magnet $\mathrm{Ho}_{2} \mathrm{Ti}_{2} \mathrm{O}_{7}$, J. Appl. Phys. 87, 5914 (2000).

[62] M. Ruminy, E. Pomjakushina, K. Iida, K. Kamazawa, D. T. Adroja, U. Stuhr, and T. Fennell, Crystal-Field Parameters of the Rare-Earth Pyrochlores $R_{2} \mathrm{Ti}_{2} \mathrm{O}_{7}(R=\mathrm{Tb}, D y$, and Ho), Phys. Rev. B 94, 024430 (2016).

[63] J. G. Rau and M. J. P. Gingras, Magnitude of Quantum Effects in Classical Spin Ices, Phys. Rev. B 92, 144417 (2015).

[64] A. Abragam and B. Bleaney, Electron Paramagnetic Resonance of Transition Ions (Oxford University Press, Oxford, 2012).

[65] M. Krusius, A. C. Anderson, and B. Holmström, Calorimetric Investigation of Hyperfine Interactions in Metallic Ho and Tb, Phys. Rev. 177, 910 (1969).

[66] J. Kondo, Internal Magnetic Field in Rare Earth Metals, J. Phys. Soc. Jpn. 16, 1690 (1961).

[67] R. Brout, K. A. Müller, and H. Thomas, Tunnelling and Collective Excitations in a Microscopic Model of Ferroelectricity, Solid State Commun. 4, 507 (1966).

[68] R. B. Stinchcombe, Ising Model in a Transverse Field. I. Basic Theory, J. Phys. C 6, 2459 (1973).

[69] P. W. Anderson, Random-Phase Approximation in the Theory of Superconductivity, Phys. Rev. 112, 1900 (1958).

[70] S. Suzuki, J.-I. Inoue, and B. K. Chakrabarti, Quantum Ising Phases and Transitions in Transverse Ising Models, 2nd ed. (Springer, Heidelberg, 2012).

[71] A. Dutta, G. Aeppli, B. K. Chakrabarti, U. Divakaran, T. F. Rosenbaum, and D. Sen, Quantum Phase Transitions in
Transverse Field Spin Models: From Statistical Physics to Quantum Information (Cambridge University Press, Cambridge, England 2015).

[72] H. M. Rønnow, R. Parthasarathy, J. Jensen, G. Aeppli, T. F. Rosenbaum, and D. F. McMorrow, Quantum Phase Transition of a Magnet in a Spin Bath, Science 308, 389 (2005).

[73] R. Coldea, D. A. Tennant, E. M. Wheeler, E. Wawrzynska, D. Prabhakaran, M. Telling, K. Habicht, P. Smeibidl, and K. Kiefer, Quantum Criticality in an Ising Chain: Experimental Evidence for Emergent $\mathrm{E}_{8}$ Symmetry, Science 327, 177 (2010).

[74] B. Tomasello, C. Castelnovo, R. Moessner, and J. Quintanilla, Correlated Quantum Tunneling of Monopoles in Spin Ice, Phys. Rev. Lett. 123, 067204 (2019).

[75] P. Bonville, I. Mirebeau, A. Gukasov, S. Petit, and J. Robert, Tetragonal Distortion Yielding a Two-Singlet Spin Liquid in Pyrochlore $\mathrm{Tb}_{2} \mathrm{Ti}_{2} \mathrm{O}_{7}$, Phys. Rev. B 84, 184409 (2011).

[76] S. Petit, P. Bonville, J. Robert, C. Decorse, and I. Mirebeau, Spin Liquid Correlations, Anisotropic Exchange, and Symmetry Breaking in $\mathrm{Tb}_{2} \mathrm{Ti}_{2} \mathrm{O}_{7}$, Phys. Rev. B 86, 174403 (2012).

[77] O. Benton, Instabilities of a U(1) Quantum Spin Liquid in Disordered Non-Kramers Pyrochlores, Phys. Rev. Lett. 121, 037203 (2018).

[78] S. T. Bramwell, M. J. Harris, B. C. den Hertog, M. J. P. Gingras, J. S. Gardner, D. F. McMorrow, A. R. Wildes, A. Cornelius, J. D. M. Champion, R. G. Melko, and T. Fennell, Spin Correlations in $\mathrm{Ho}_{2} \mathrm{Ti}_{2} \mathrm{O}_{7}$ : A Dipolar Spin Ice System, Phys. Rev. Lett. 87, 047205 (2001).

[79] G. Mennenga, L. J. de Jongh, and W. J. Huiskamp, Field Dependent Specific Heat Study of the Dipolar Ising Ferromagnet $\mathrm{LiHoF}_{4}$, J. Magn. Magn. Mater. 44, 59 (1984).

[80] A. P. Ramirez and J. Jensen, The Nuclear Heat Capacity of $\mathrm{HoF}_{3}$, J. Phys. Condens. Matter 6, L215 (1994).

[81] T. N. Tommet and D. L. Huber, Dynamics of the Longitudinal Spin for the Three-Dimensional, Spin-1/2 Ising Model in a Transverse Field, Phys. Rev. B 11, 1971 (1975).

[82] J. Oitmaa, M. Plischke, and T. A. Winchester, HighTemperature Dynamics of the Ising Model in a Transverse Field, Phys. Rev. B 29, 1321 (1984).

[83] J. Florencio, Jr., S. Sen, and Z.-X. Cai, Dynamic Structure Factor of the Transverse Ising Model, J. Phys. Condens. Matter 7, 1363 (1995).

[84] J. Kotzler, H. Neuhaus-Steinmetz, A. Froese, and D. Gorlitz, Relaxation-Coupled Order-Parameter Oscillation in a Transverse Ising System, Phys. Rev. Lett. 60, 647 (1988).

[85] R. W. Youngblood, G. Aeppli, J. D. Axe, and J. A. Griffin, Spin Dynamics of a Model Singlet Ground-State System, Phys. Rev. Lett. 49, 1724 (1982).

[86] R. G. Lloyd and P. W. Mitchell, Spin Dynamics in the Singlet-Singlet Ground State Magnet $\mathrm{LiTb}_{x} \mathrm{Y}_{1-x} \mathrm{~F}_{4}: A$ Neutron Scattering Study, J. Phys. Condens. Matter 2, 2383 (1990).

[87] J. A. M. Paddison, P. Mukherjee, X. Bai, Z. L. Dun, C. R. Wiebe, H. D. Zhou, J.S. Gardner, M. Mourigal, and S. Dutton, Modeling Spin Dynamics in the Singlet Ground State Garnet $\mathrm{Ho}_{3} \mathrm{Ga}_{5} \mathrm{O}_{12}$, arXiv:1908.03530. 
[88] V. H. Santos and C. Scherer, A New Approximation for the Dynamics of the Transverse Ising Model, Z. Phys. B 40, 95 (1980).

[89] M. Enjalran and M. J. P. Gingras, Theory of Paramagnetic Scattering in Highly Frustrated Magnets with Long-Range Dipole-Dipole Interactions: The Case of the $\mathrm{Tb}_{2} \mathrm{Ti}_{2} \mathrm{O}_{7}$ Pyrochlore Antiferromagnet, Phys. Rev. B 70, 174426 (2004).

[90] G. Ehlers, A. L. Cornelius, M. Orendác, M. Kajnaková, T. Fennell, S. T. Bramwell, and J. S. Gardner, Dynamical Crossover in 'Hot' Spin Ice, J. Phys. Condens. Matter 15, L9 (2003).

[91] O. Benton, Quantum Origins of Moment Fragmentation in $\mathrm{Nd}_{2} \mathrm{Zr}_{2} \mathrm{O}_{7}$, Phys. Rev. B 94, 104430 (2016).

[92] O. Cépas, Colorings of Odd or Even Chirality on Hexagonal Lattices, Phys. Rev. B 95, 064405 (2017).

[93] M. D. Núñez Regueiro, C. Lacroix, and R. Ballou, Effect of Frustration Near the Magnetic-Nonmagnetic Transition, Phys. Rev. B 46, 990 (1992).

[94] R. M. Nicklow, R. M. Moon, S. Kawarazaki, N. Kunitomi, H. Suzuki, T. Ohtsuka, and Y. Morii, Nuclear Spin Ordering Observed by Neutron Diffraction, J. Appl. Phys. 57, 3784 (1985).

[95] A. R. Mackintosh, Rare Earth Magnetism: Structures and Excitations (Clarendon Press, Oxford, 1991).

[96] C. Paulsen, S. R. Gilbin, E. Lhotel, D. Prabhakaran, K. Matsuhira, G. Balakrishnan, and S. T. Bramwell, Nuclear Spin Assisted Quantum Tunnelling of Magnetic Monopoles in Spin Ice, Nat. Commun. 10, 1509 (2019).

[97] K. Kimura, S. Nakatsuji, J-J. Wen, C. Broholm, M. B. Stone, E. Nishibori, and H. Sawa, Quantum Fluctuations in Spin-Ice-like $\operatorname{Pr}_{2} \mathrm{Zr}_{2} \mathrm{O}_{7}$, Nat. Commun. 4, 1934 (2013).

[98] http://energy.gov/downloads/doe-public-access-plan.
[99] O. V. Kovalev, Representations of the Crystallographic Space Groups, (Gordon and Breach Science Publishers, Amsterdam, 1993).

[100] U. Walter, Treating Crystal Field Parameters in Lower than Cubic Symmetries, J. Phys. Chem. Solids 45, 401 (1984).

[101] J. J. Baldoví, S. Cardona-Serra, J. M. Clemente-Juan, E. Coronado, A. Gaita-Ariño, and A. Palii, SIMPRE: A Software Package to Calculate Crystal Field Parameters, Energy Levels, and Magnetic Properties on Mononuclear Lanthanoid Complexes Based on Charge Distributions, J. Comput. Chem. 34, 1961 (2013).

[102] A. Scheie, M. Sanders, J. Krizan, A. D. Christianson, V. O. Garlea, R. J. Cava, and C. Broholm, Crystal Field Levels and Magnetic Anisotropy in the Kagome Compounds $\mathrm{Nd}_{3} \mathrm{Sb}_{3} \mathrm{Mg}_{2} \mathrm{O}_{14}, \quad \mathrm{Nd}_{3} \mathrm{Sb}_{3} \mathrm{Zn}_{2} \mathrm{O}_{14}$, and $\mathrm{Pr}_{3} \mathrm{Sb}_{3} \mathrm{Mg}_{2} \mathrm{O}_{14}$, Phys. Rev. B 98, 134401 (2018).

[103] G. Shirane and J. D. Axe, Acoustic-Phonon Instability and Critical Scattering in $\mathrm{Nb}_{3} \mathrm{Sb}$, Phys. Rev. Lett. 27, 1803 (1971).

[104] P. J. Brown, International Tables for Crystallography (Kluwer Academic Publishers, Dordrecht, 2004), pp. 454-460.

[105] S. W. de Leeuw, J. W. Perram, and E. R. Smith, Simulation of Electrostatic Systems in Periodic Boundary Conditions. II. Equivalence of Boundary Conditions, Proc. R. Soc. A 373, 57 (1980).

[106] R. G. Melko and M. J. P. Gingras, Monte Carlo Studies of the Dipolar Spin Ice Model, J. Phys. Condens. Matter 16, R1277 (2004).

[107] A. Aguado and P. A. Madden, Ewald Summation of Electrostatic Multipole Interactions Up to the Quadrupolar Level, J. Chem. Phys. 119, 7471 (2003). 